$\mathrm{DOE} / \mathrm{NV} / 10630--28-$ Add .1

\title{
ADDENDUM TO ENVIRONMENTAL MONITORING PLAN NEVADA TEST SITE AND SUPPORT FACILITIES
}

November 1992

Work Performed Under

Contract No. DE-AC08-89NV10630

Prepared for the

U. S. Department of Energy

Nevada Field Office

Prepared by:

Reynolds Electrical \& Engineering Co., Inc.

Post Office Box 98521

Las Vegas, Nevada 89193-8521

\section{MASTER}

DISTRIBUTION OF THIS DOCUMENT IS UNLIMITED 


\section{FOREWORD}

This 1992 Addendum to the "Environmental Monitoring Plan Nevada Test Site and Support Facilities - 1991," Report No. DOE/NV/10630-28 (EMP) applies to the U.S. Deppartment of Energy's (DOE's) operations on the Continental U.S. (including Amchitka Island, Alaska) that are under the purview of the DOE Nevada Field Office (DOE/NV). The primary F rpose of these operations is the conduct of the nuclear weapons testing program for the DOE and the Department of Defense. Since 1951, these tests have been conducted principally at the Nevada Test Site (NTS), which is located approximately 100 miles northwest of Las Vegas, Nevada. In accordance with DOE Order 5400.1, this 1992 Addendum to the EMP brings together, in one document, updated information and/or new sections to the description of the environmental activities conducted at the NTS by user organizations, operations support contractors, and the U.S. Environmental Protection Agency (EPA) originally published in the EMP. The EPA conducts both the offsite environmental monitoring program around the NTS and post-operational monitoring efforts at non-NTS test locations used between 1961 and 1973 in other parts of the continental U.S. All of these monitoring activities are conducted under the auspices of the DOE/NV, which has the stated policy of conducting its operations in compliance with both the letter and the spirit of applicable environmental statutes, regulations, and standards. 


\section{TABLE OF CONTENTS}

\section{Part I Overview}

1.0 Introduction ............................

1.1 Operations and Facilities $\ldots \ldots \ldots \ldots \ldots \ldots \ldots \ldots \ldots \ldots$ I-1

1.1.1 Operational Test Areas . . . . . . . . . . . . . . . . . I-1

1.1.1.1 Nevada Test Site Operations ............ I-1

2.0 Sources and Effluents $\ldots \ldots \ldots \ldots \ldots \ldots \ldots \ldots \ldots \ldots \ldots$ I-1

2.3 Off-NTS Continental U.S. Test Areas . . . . . . . . . . . . . . I-1

9.0 Summary of Operational Area Monitoring Plans . . . . . . . . . . . I-1

9.2 Lawrence Livermore National Laboratory Testing Areas

and Facilities, NTS . . . . . . . . . . . . . . . . . I-1

9.7 NTS/Tonopah Test Range Nonradiological Effluent Monitoring, Reynolds Electrical \& Engineering Co., Inc. . . . . . . . 1-2

9.8 Waste Disposal and Waste Management Facilities,

Reynolds Electrical \& Engineering Co., Inc., NTS . . . . . . . . . I-2

Part II.1 Operational Area Monitoring Plan for the Los Alamos National

Laboratory Testing Areas and Facilities Nevada Test Site

3.0 Effluent Monitoring Plan . . . . . . . . . . . . . . . . II.1-1

3.3 Effluent Monitoring System Design $\ldots \ldots \ldots \ldots \ldots$. . . . . . . .

3.3.1 Drillback Activities ................. II.1-1

Part II.2 Operational Area Monitoring Plan for the Lawrence Livermore National

Laboratory Testing Areas and Facilities Nevada Test Site

1.0 Introduction . . . . . . . . . . . . . . . . . . . . . . . $11.2-1$

1.1 Organizational Structure and Responsibility . . . . . . . . . . . II.2-1

3.0 Effluent Monitoring Plan . . . . . . . . . . . . . . . . . . . $11.2-1$

3.1 .2 Post-Event Monitoring . . . . . . . . . . . . . . . II.2-1

Part II.3 Operational Area Monitoring Plan for the Defense Nuclear

Agency/Sandia Testing Areas and Facilities Nevada Test Site

3.0 Effluent Monitoring Plan ... . . . . . . . . . . . . . . . . . $11.3-1$

3.2.2 Permit Requirements $\ldots \ldots \ldots \ldots \ldots \ldots \ldots \ldots \ldots$ II. 1

Part II.4 Operational Area Monitoring Plan for the Liquefied Gaseous Fuels Spill

Test Facility Nevada Test Site

2.0 Effluents . . . . . . . . . . . . . . . . . . . $11.4-1$

2.1 Inventory of Effluents $\ldots \ldots \ldots \ldots \ldots \ldots \ldots \ldots \ldots \ldots$ II.4-1

Fart II.5A Operational Area Monitoring Plan for the Onsite Radiological

Surveillance Program Nevada Test Site

2.0 Effluents (Emissions and Discharges) . . . . . . . . . . . II.5A-1

2.1.2 Fladioactive Liquid Discharges . . . . . . . . . . II.5A-1

3.0 Effluent Monitoring Plan . . . . . . . . . . . . . . . . 11.5 A-1

3.2.1 Liquid Discharges . . . . . . . . . . . . . . . . $11.5 \mathrm{~A}-1$

3.2.2.5 Area 25 Treatability Test Facility . . . . . . . . II.5A-1

5.0 Analytical Procedures . . . . . . . . . . . . . . . . II.5A-2

5.1 Analyses Employed $\ldots \ldots \ldots \ldots \ldots \ldots \ldots \ldots \ldots$ II.5A-2 
Part II.5B Operational Area Monitoring Plan for Nonradiological Monitoring at the Tonopah Test Range and Nevada Test Site

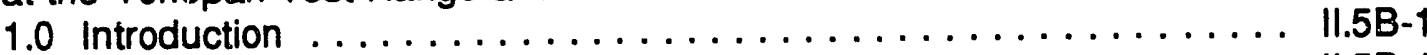

1.2 Operational Activities $\ldots \ldots \ldots \ldots \ldots \ldots \ldots \ldots \ldots \ldots \ldots \ldots \ldots$ II.5B-1

2.0 Rationale ............................ II.5B-1

2.1 Liquid Discharges . . . . . . . . . . . . . . . . II.5B-1

2.1.1 Domestic Sewage . . . . . . . . . . . . II.5B-1

2.1 .2 Injection Wells .................... II.5B-2

2.2 Air Emissions . . . . . . . . . . . . . . . . . . . II.5B-2

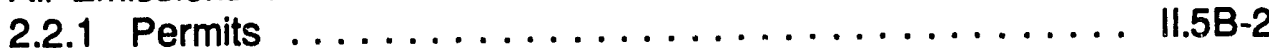

2.7 Miscellaneous Sampling . . . . . . . . . . . . . . . II.5B-2

2.8 Ecological Monitoring ... . . . . . . . . . . . . II.5B-2

3.0 Effluents (Emissions and Discharges) . . . . . . . . . . . . II.5B-3

3.1 Monitoring System Design - Water and Wastewater . . . . . . . II.5B-3

3.1.1 Drinking Water ..................... II.5B-3

3.2 Monitoring System Design - Air Emissions . . . . . . . . . . II.5B-3

3.2.1 Air Emissions . . . . . . . . . . . . . . . . . II.5B-3

4.0 Environmental Surveillance . . . . . . . . . . . . . . . . . II.5B-4

4.1 Standard Operating Procedures/Implementing Procedures . . . II.5B-4

Part II.5C Operational Area Monitoring Plan for the Waste Management Department Nevada Test Site

2.0 Effluents (Emissions and Discharges) $\ldots \ldots \ldots \ldots \ldots \ldots \ldots$. . . . . . . .

4.0 Environmental Surveillance Plan . . . . . . . . . . . . . . . II.5C-1

4.1 Meteorological Monitoring . . . . . . . . . . . . . II.5C-1

4.2 Ongoing Waste Management Studies ............. . . .5C-1

4.3 Surveillance Description . . . . . . . . . . . . . II.5C-1

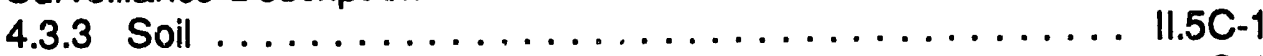

4.3.6 Vadose Zone . . . . . . . . . . . . . . . . II.5C-1

9.0 Public Dose Assessment . . . . . . . . . . . . . . . . . II.5C-2

Part II.6 Operational Area Monitoring Plan for Test Support Services Nevada

Test Site

1.0 Introduction $\ldots \ldots \ldots \ldots \ldots \ldots \ldots \ldots \ldots \ldots \ldots \ldots \ldots . \ldots \ldots$. $\ldots \ldots \ldots$

2.0 Effluents (Emissions and Discharges) $\ldots \ldots \ldots \ldots \ldots \ldots \ldots$. . . . . . . .

2.1 NTS Facilities . . . . . . . . . . . . . . . . . . . . II.6-1

2.1.2 Inventory of Surface Water Discharges ........... II.6-1

2.2 NV Support Facilities . . . . . . . . . . . . . . . . . II.6-1

2.2.2 Inveniory of Liquid Discharges . . . . . . . . . II.6-1

3.0 Effluent Monitoring Plan ...................... II.6-1

3.2 Effluent Monitoring Design Criteria . . . . . . . . . . . . . . II.6-1

3.2.3 Spent Photographic Solutions from Las Vegas Facilities .. II.6-1

6.0 Quality Assurance and Quality Control ... . . . . . . . . . . . II.6-2

Part II.7A Operational Area Monitoring Plan for the Offsite Radiological Safety Program Nevada Test Site

4.0 Environmental Surveillance Plan . . . . . . . . . . . . . . . . II.7A-1

4.1 Rationale ........................ . . . . . . .

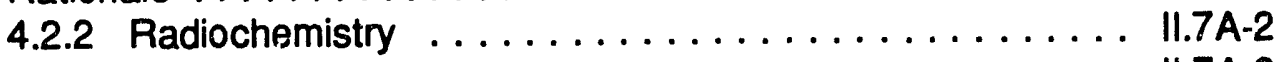

4.3 Network Design ...................... II.7A-2 
4.3.1 Sample Collection Locations . . . . . . . . . . . II.7A-2

4.3.5 Pressurized Ion Chamber Network . . . . . . . . $11.7 A-2$

4.3.10 Hydrological Monitoring Program . . . . . . . . . $11.7 A-2$

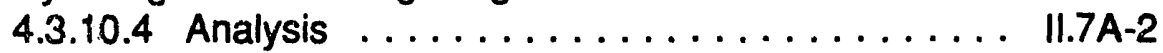

8.0 Data Management . . . . . . . . . . . . . . . . . II.7A-3

8.1 Radiochemical Database . . . . . . . . . . . . . . . II.7A-3

8.2 Thermoluminescent Dosimetry Database . . . . . . . . . . . II.7A-3

8.3 Internal Dosimetry Database . . . . . . . . . . . . . . . II.7A-4

8.4 Pressurized Ion Chamber Database . . . . . . . . . . . . . . II.7A-4

Part II.7B Operational Area Monitoring Plan for the Long-Term Hydrological

Monitoring Program Off The Nevada Test Site

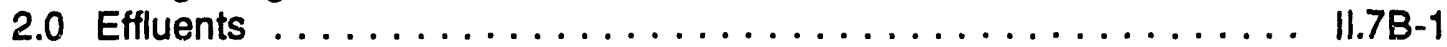

4.0 Environmiental Surveillance Plan . . . . . . . . . . . . . . . II.7B-1

4.2 Design Criteria . . . . . . . . . . . . . . . . . II.7B-1

4.2.2 Data Quality Objectives ............... II.7B-1

4.3 Amchitka Island Projects, Alaska . . . . . . . . . . . . . II.7B-2

4.3.3.2 Surveillance System Design . . . . . . . . . II.7B-2

4.6 Project DRIBBLE, Mississippi . . . . . . . . . . . . . II.7B-2

4.6.3 Environmental Surveillance . . . . . . . . . . II.7B-2

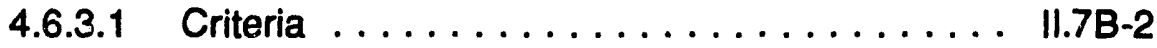

4.8 Project SHOAL, Nevada . . . . . . . . . . . . . . . . . II.7B-2

4.9 Project GASBUGGY, New Mexico . . . . . . . . . . . . . II.7B-2

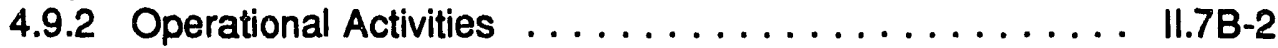

7.0 Data Management . . . . . . . . . . . . . . . . II.7B-3

Part II.8 Operational Area Monitoring Plan for EG\&G/Energy Measurements, Inc.

Support Facilities for the Nevada Test Site

3.0 Effluents and Monitoring Plan . . . . . . . . . . . . . . . . II.8-1

3.1 Monitoring Requirements for EG\&G/EM Operations . . . . . . . . II.8-1

3.1.2 Effluent Monitoring Design Criteria . . . . . . . . . . II.8-1

3.1.2.1 Standards, Criteria, and Action Levels . . . . . . II.8-1

3.2.2 Monitoring Design Criteria . . . . . . . . . . . . II.8-1

3.2.3.1 Wastewater Monitoring Systems . . . . . . . II.8-1

3.3 Amador Valley Operations . . . . . . . . . . . . . . . . II.8-1

3.3.2 Monitoring Design Criteria . . . . . . . . . . . . II.8-1

3.3.3 Effluent Monitoring System Design . . . . . . . . . . II.8-2

3.4 Kirtland Operations . . . . . . . . . . . . . . . . . . II.8-2

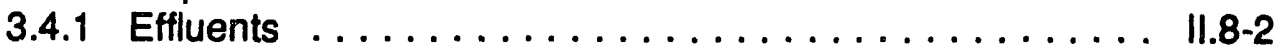

3.4.2 Monitoring Design Criteria . . . . . . . . . . . . . II.8-2

3.4.3 Effluent Monitoring System Design . . . . . . . . . . $11.8-2$

3.6 Santa Barbara Operations . . . . . . . . . . . . . . . . . . II.8-2

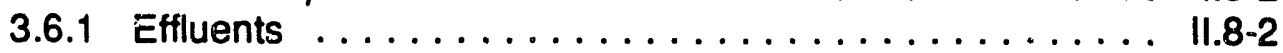

3.6.2 Effluent Monitoring Design Criteria . . . . . . . . . . II.8-2

3.6.3 Effluent Monitoring System Design . . . . . . . . . . $11.8-3$

3.7 Special Technologies Laboratory . . . . . . . . . . . . . $11.8-3$

3.7.1 Effluents ......................

3.7.2 Effluent Monitoring Design Criteria . . . . . . . . . . II.8-3

3.7.3 Effluent Monitoring System Design . . . . . . . . II.8-3

3.9 Wobum Cathode Ray Tube Operation . . . . . . . . . . II.8-3 
3.9.3 Effluent Monitoring System Design . . . . . . . . II.8-3

6.0 Quality Assurance and Quality Control . . . . . . . . . . . . . . II.8-4

6.1 Quality Assurance Management . . . . . . . . . . . . . 11.8-4

6.2 Data Management and Reports . . . . . . . . . . . . . . . II.8-4

6.3 QA Program Assessment . . . . . . . . . . . . . . . $11.8-4$

6.4 Personnel Qualifications and Training ... . . . . . . . . II.8-5

6.5 Laboratory Services . . . . . . . . . . . . . . . . $11.8-5$

6.6 Data Collection and Management ... . . . . . . . . . II.8-5

6.6.1 Sample Point Selection . . . . . . . . . . . . . . II.8-5

6.6.2 Sampling Method . . . . . . . . . . . . . . . II.8-5

6.6.2.1 Sampling Frequency $\ldots \ldots \ldots \ldots \ldots \ldots \ldots$. $\ldots \ldots .8-5$

6.6.2.2 Type of Sample . . . . . . . . . . . . . II.8-5

6.6.2.3 Sample Management . . . . . . . . . . . $11.8-6$

6.6.2.4 Wastewater Sampling Equipment . . . . . . . . II.8-7

6.6.2.5 Quality Control Procedures for Sampling . . . . . II.8-7

6.6.3 Documentation of Samples and Sampling Activity . . . . II.8-7

6.6.3.1 Sample Container Labels . . . . . . . . . . . II.8-7

6.6.3.2 Sarnple Container Seals . . . . . . . . . . II.8-8

6.6.3.3 Sample Logs . . . . . . . . . . . . . . . . . . $11.8-8$

6.6.3.4 Chain of Custody Record and Procedures ... . . II.8-8

6.6.4 Laboratory Practice Analysis and Certification ........ II.8-9

6.6.4.1 Laboratory Practice . . . . . . . . . . . . . II.8-9

6.6.4.2 Analytical Procedure .............. II.8-9

6.6.4.3 Laboratory Certification and QAVQC Programs . . II.8-9

6.6.5 Air Emission Calculations . . . . . . . . . . . . . II.8-10

6.7 Discrepancies and Corrective Action . . . . . . . . . . . . . . II.8-10

6.8 Independent Data Verification . . . . . . . . . . . . II.8-10

Part III Groundwater Monitoring Plan for the Nevada Test Site

1.0 introduction ........................... III-1

1.1 Groundwater Protection Management Plan . . . . . . . . . . . . III-1

Part IV Meteorological Monitoring Plan of the Weather Service Nuclear Support Office for the Nevada Test Site

2.0 Meteorological Monitoring . . . . . . . . . . . . . . . IV-1

2.3 National Weather Service Systems ................. IV

2.3.2 Satellite Weather Information System . . . . . . . . IV-1

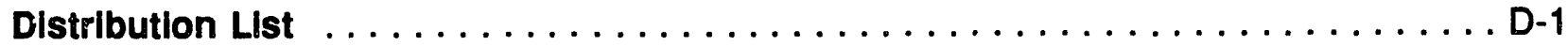


Table of Contents, cont.

\section{List of Tables}

Page

Table 1-1 List of Approved Chemicals . . . . . . . . . . . . . . . . . . . . II.4-1

Table 4-1 Summary of the Environmental Surveillance Program . . . . . . . . . II.5C-3

\section{List of Exhiblts}

Exhibit 3-1 LANL RAMS Array for Nuclear Tests . . . . . . . . . . . . . . . II.1-2

Exhibit 4-1 Potential Exposure Pathways Diagram . . . . . . . . . . . . . . . . II.5A-3

Exhibit 4-6 Summary of the NTS Radiological Surveillance Program . . . . . . . . . II.5A-4

Exhibit 5-1 Summary of Laboratory Analytical Procedures . . . . . . . . . . . . II.5A-5

\section{Appendicies}

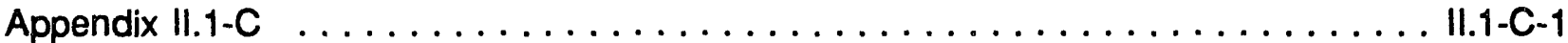

Appendix II.1-D . . . . . . . . . . . . . . . . . . . . . . II.1-D-1

\section{Feferences}

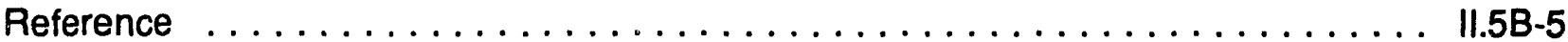




\section{SECTION I}

\section{OVERVIEW}

by

A. R. Latham

Reynolds Electrical \& Engineering Co., Inc.

January 21, 1993 


\subsection{Introduction}

\subsection{Operations and Facilities}

\subsubsection{Operational Test Areas}

\subsubsection{Nevada Test Site Operations}

Nuclear testing at the NTS is currently conducted in three areas: at the bottom of plugged vertical shafts drilled into (1) Yucca Flat or into (2) Pahute Mesa and in sealed tunnels mined into (3) Rainier Mesa. Nuclear safety tests were conducted in the 1950s and 1960s on the Range Complex and on the TTR. Low-level radioactive waste disposal (burial) facilities are located in Frenchman Flat and Yucca Flat. Transuranic waste is containerized and stored in Frenchman Flat pending shipment to the Waste Isolation Pilot Plant in New Mexico. Other testing facilities on the NTS include the (non-nuclear) Liquified Gaseous Fuels Spill Test Facility (LGFSTF) in Frenchman Flat, the Treatability Test Facility located in Area 25, and the Nevada Research and Development Area in Jackass Flats. Construction and maintenance facilities consisting of offices, shops, laboratories, and worker housing facilities are located at the NTS base camp at Mercury and the Area 12 Camp. Other construction and maintenance facilities are located in Area 3, Area 20, and at the NTS Control Point. The current operational areas and facilities are shown in Exhibit 1-2. Nuclear testing on the NTS is conducted by the NTS user organizations; Lawrence Livermore National Laboratory (LLNL), Los Alamos National Laboratory (LANL), and Defense Nuclear Agency (DNA). Private sector firms or consortiums conduct non-nuclear liquid spill tests at the LGFSTF.

\subsection{Sources and Effluents}

\subsection{Off-NTS Continental U.S. Test Areas}

No effluents are produced at these sites. At most sites, contamination is confined to the residual radioactivity remaining at the point of the underground nuclear explosion. Accidental leakage caused low levels of tritium contamination at the surface on Amchitka Island at the LONG SHOT site, and waste disposal resulted in low surface levels of tritium at the Project DRIBBLE site in Mississippi, but only residual contamination (tritium) remains at both sites. Since 1984, a monitoring well near the project GASBUGGY ground zero (GZ) has indicated slightly elevated levels of tritium; however, these levels are well below the limits set in the Interim Primary Drinking Water Regulations.

\subsection{Summary of Operational Area Monitoring Plans}

\subsection{Lawrence Livermore National Laboratory Testing Areas and Facilities, NTS}

This OAMP describes effluent monitoring plans for underground nuclear tests conducted in plugged vertical shafts orilled into LLNL testing areas located in Areas 2, 4, 8, 9, 10, 12, and 20 of the NTS. The Laboratory conducts no ambient environmental surveillance monitoring at 
the NTS. LLNL effluent monitoring is for radioactive gaseous and particulate emissions to the atmosphere from venting, seepage, device assembly, and post-test ("post-shot") drillback operations conducted to collect samples or equipment from the vicinity of the underground test. Liquids and/or drilling muds from drilling operations are sampled at time of discharge into tanks. There are no liquid or mud discharges to surface drainage channels or to the offsite environment. Environmental surveillance in the LLNL areas is performed by REECo.

\subsection{NTS/Tonopah Test Range Nonradiological Effluent Monitoring, Reynolds Electrical \& Engineering Co., Inc.}

This plan contains a listing of the sources of air and wastewater discharges on the NTS, the permits required from the state of Nevada for operation of the sources, and the monitoring required to comply with the permits. Other monitoring is conducted to comply with the requirements of federal and state statutes. Ambient air quality and stationary source monitoring has been performed to assess the compliance with federal, state, and local regulations.

\subsection{Waste Disposal and Waste Management Facilities, Reynolds Electrical \& Engineering Co., Inc., NTS}

This plan covers monitoring of the two sites on the NTS used for the disposal of radioactive low-level waste (LLW). The site in Area 3 consists of two adjoining surface subsidence craters used for the disposal of bulk LLW. The site in Area 5 includes facilities for various kinds of waste management such as burial of LLW, greater confinement disposal (deep borehole) of other LLW, a transuranic waste storage cell (pending shipment to the Waste Isolation Pilot Plant in New Mexico), a mixed waste management unit (Rocky Flats waste only), and a storage area for accumulation of hazardous waste that is shipped to non-NTS RCRA-permitted commercial disposal facilities every 90 days. The OAMP describes the surveillance plan as presently designed and a vadose monitoring procedure that is being considered. The RWMS pits in Area 5 are being studied to determine if them should be classified as diffuse air sources. 


\section{SECTION II.1}

\section{Operational Area Monitoring Plan for the Los Alamos National Laboratory Testing Areas and Facilities \\ Nevada Test Site}




\subsection{Effluent Monitoring Plan}

\subsection{Effluent Monitoring System Design}

\subsubsection{Drilliback Activities}

The monitoring for each drillback activity is done by use of several devices as shown in Exhibits 3-2 and 3-3, one of which is a constant monitoring system. The air in the system is continuously monitored for gamma-emitting radionuclides. This system is calibrated to respond to gamma energies above $80 \mathrm{keV}$, the predominant transition energy of ${ }^{133} \mathrm{Xe}$, which is the most abundant nuclide in the gas mix seen at the surface during most drillback operations. This radionuclide acts as an early warning, indicating there is material in the stack, and allows for remedial action before a release occurs. Detailed information is in LANL-NTS-DP-002 (Appendix II.1-C). The monitoring of any release from the cellar during the drillback or cementback phases is done by a flow through system sampling the Auxiliary Cellar Exhaust Blower. A sample of the exhausted air is drawn through the system, and analyzed for ${ }^{133} \mathrm{Xe}$ in the Rad Lab. Detailed information is given in Draft Procedure, LANLNTS-DP-001 (Appendix II.1-D). 


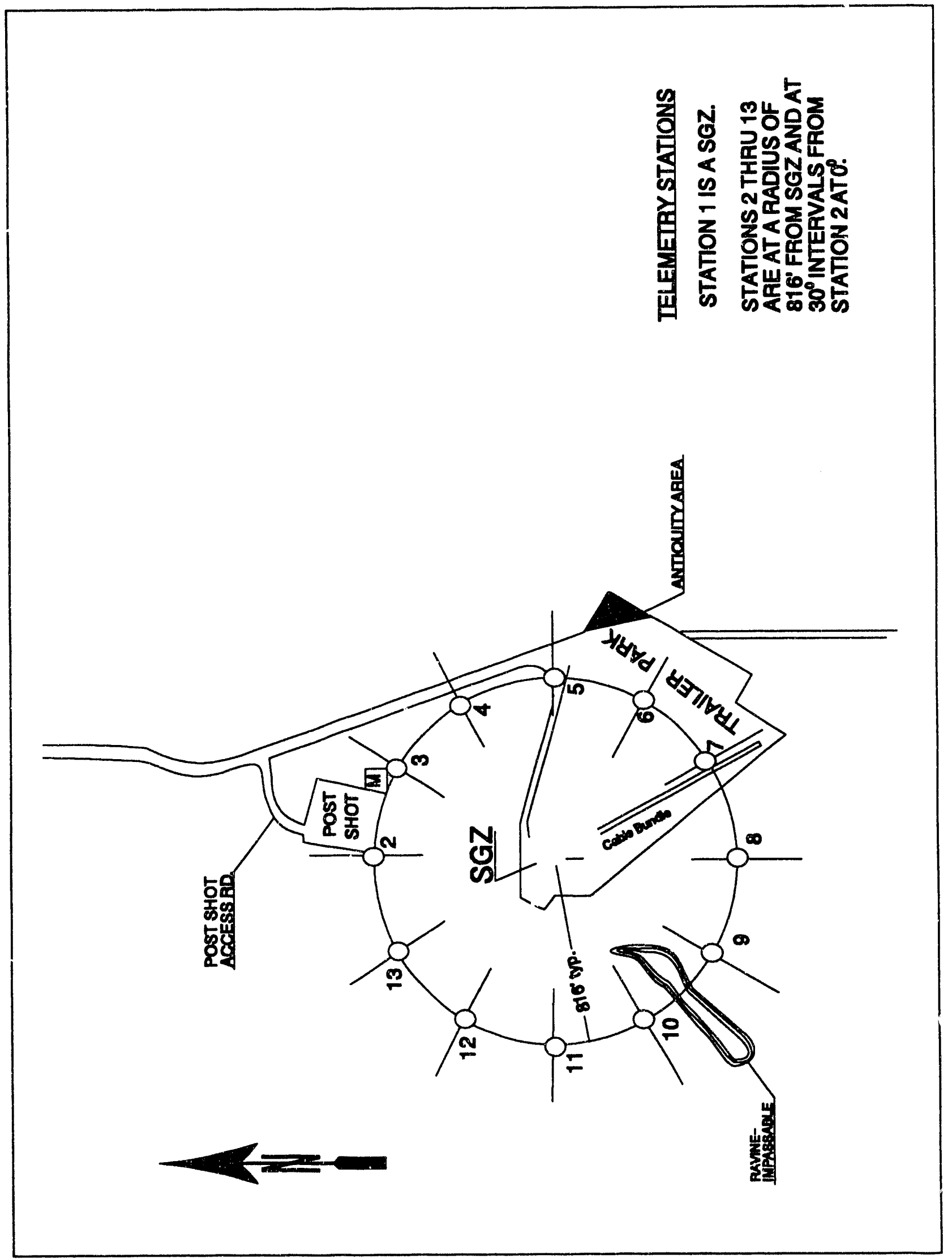

Exhibit 3-1 LANL RAMS Array for Nuclear Tests 


\section{APPENDIX II.1-C}

\section{DETAILED PROCEDURE FOR ESTIMATION OF ACTIVITY IN THE CONTAINMENT SYSTEM}




\section{DRAFT LANL-NTS-DP-002, RO Page 1 of 3 \\ EIELD TEST HEALTH PHYSICS SECTION DETAILED PROCEDURE FOR \\ ESTIMATION OF ACTIVITY IN THE CONTAINMENT SYSTEM}

I. PURPOSE

To document the procedures used for the estimation of quantities of radioactive material inside the containment system during drillback operations.

II. SCOPE

This procedure applies to the interpretation of results derived from the radio-metric analysis of a sample of the air drawn from the containment system into the Mobile Radiological iaboratory (Rad Lab) trailer.

III. DEFINITIONS

Containment Stack or Stack The gas field hardware mounted on the top of the well casing designed to contain the radioactive and other gasses in the chimney. This is also referred to as the "blowout preventer".

Cellar Excavated and cased area below the drill rig housing the containment stack.

Rad Lab A trailer containing analytical equipment for the analysis of various of air streams. This includes a system that analyzes the air in the containment system for both radioactive gasses and combustible gasses.

Core off-gas Airborne radioactive material arising from solid core material brought to the surface during coring operations.

Chimney or Cavity Gas Gaseous material, very rich in xenon, which fills the void spaces of the cavity and the rubble chimney.

IV. DESCRIPTION Of OPERATIONS

Following most nuclear experiments on the NTS, the recovery of solid samples of debris is required. This process is referred to as a drillback. The design of the Los Alamos National Laboratory drillback hardware is to completely contain all chimney gas. Figure 1 shows the hardware as it is configured to contain the gasses. Air is drawn from the cellar, sampled by the Rad Lab, and blown down the annulus below the containment stack. 


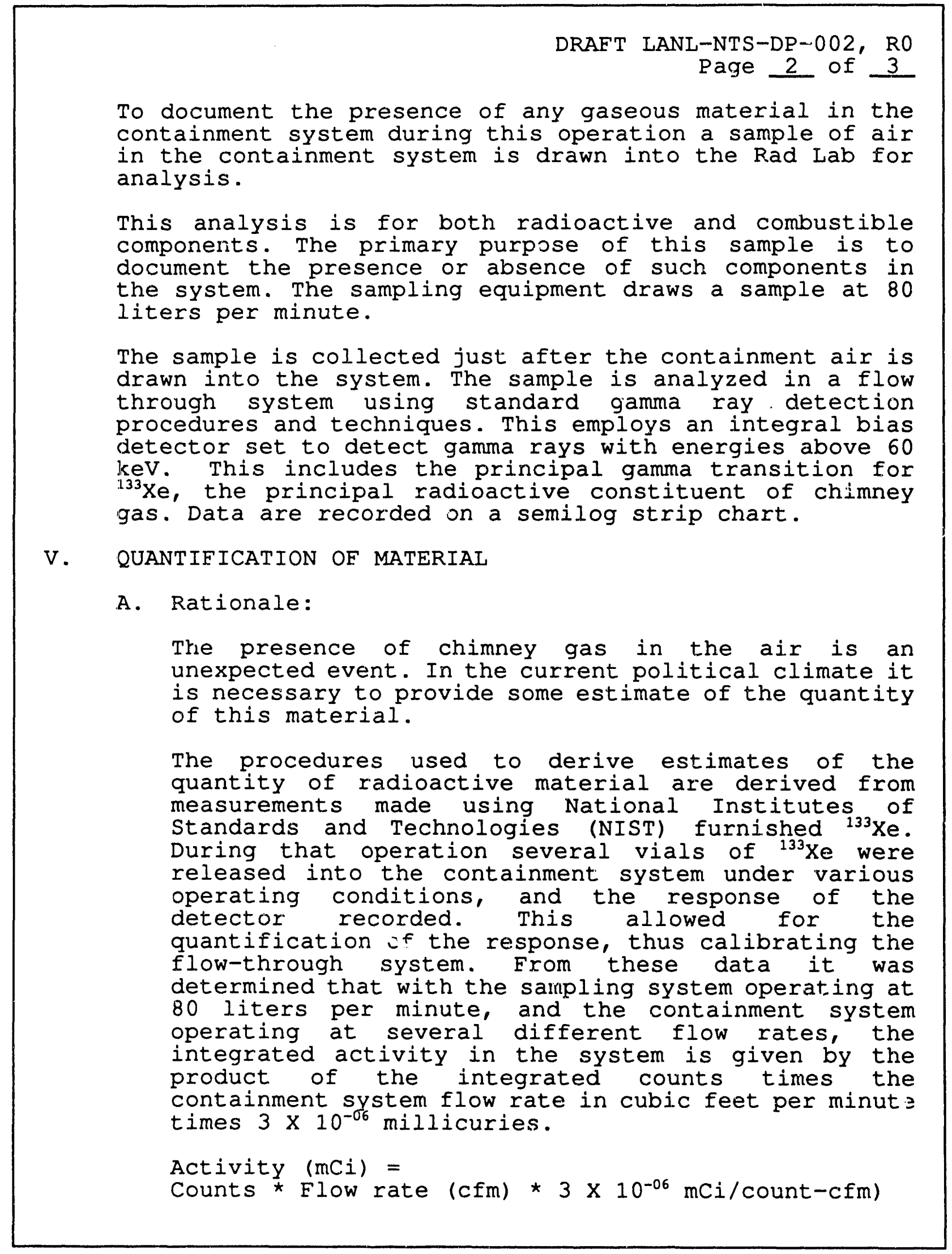




\section{DRAFT IAANL-NTS-DP-002, RO Page 3 of 3}

B. Procedure:

Once the presence of chimney gas has been verified, the integrated count of the detector system is calculated from the strip chart. The flow rate in the containment system is determined. The ${ }^{133} \mathrm{Xe}$ activity is then determined using the function given above. Under steady state conditions, the counting rate may be used to calculate the rate of material passing through the system. 


\section{APPENDIX II.1-D}

\section{DETAILED PROCEDURE FOR ESTIMATION OF ACTIVITY FROM THE AUXILIARY CELLAR EXHAUST BLOWER}




\section{FIELD TEST HEALTH PHYSICS SECTION DE'IATLED PROCEDURE FOR \\ ESTIMATION OF ACTIVITY FROM THE AUXILIARY CELLAR EXHAUST BLOWER}

I. PURPOSE

To document the procedures used for the estimation of quantities of radioactive material released during auxiliary purging of the cellar during drillback operations.

II. SCOPE

This procedure applies to the interpretation of results derived from the radio-metric analysis of a sample of the air drawn from the auxiliary cellar exhaust blower into the Mobile Radiological Laboratory (Rad Lab) trailer.

III. DEFINITIONS

Auxiliary Exhaust Blower $A$ blower and duct system designed to remove explosive mixtures from the cellar.

Cellar Excavated and cased area below the drill rig housing the containment stack.

Rad Lab A trailer containing analytical equipment for the analysis of various of air streams. This includes a system that analyses the air in the containment system for both radioactive gasses and combustible gasses.

Chimney or Cavity Gas Gaseous material, very rich in xenon, which fills the void spaces of the cavity and the rubble chimney.

IT. DESCRIPTION OF OPERATIONS

Following most nuclear experiments on the NTS, the recovery of solid samples of debris is required. This process is referred to as a drillback. The design of the Los Alamos National Laboratory drillback hardware is to completely contain all chimney gas.

During drilling operations, chimney gas, which may contain explosive components, may escape into the cellar. Before the cellar may be entered for remedial work, these mixtures must be removed. The standard practice is to contain these mixtures and return them down the annulus. 
DRAFT LANL-NTS-DP-001, R0

Page $\underline{2}$ of $\underline{4}$

If this cannot be done in a timely manner, then the mixture will be exhausted using the auxiliary blower. The main purpose of the blower is to abate explosive gasses accumulating in the cellar from the BOP. This allows for the safe and prompt repair of the hardware in the cellar.

A sample of the exhausted air is drawn into the Rad Lab for analysis. The purpose of this sample is to document the presence or absence of ${ }^{133} \mathrm{Xe}$ in the air stream. The sampling equipment draws a sample at 80 liters per minute.

The sample is collected just before the air is exhausted from the system. The sample is analyzed in a flow through system using standard gamma ray detection procedures and techniques. This employs an integral bias detector set to detect gamma rays with energies above $60 \mathrm{keV}$. This includes the principal gamma transition for ${ }^{133} \mathrm{Xe}$, the principal radioactive constituent of chimney gas. Data are recorded on a semilog strip chart.

V. QUANTIFICATION OF MATERIAL

A. Rationale:

The release of radioactive material is an unexpected event. In the current political climate it is necessary to provide an estimate of the quantity of this material.

The procedures used to derive estimates of the quantity of radioactive material are derived from measurements made using National Institutes of Standards and Technologies (NIST) furnished ${ }^{133} \mathrm{Xe}$. During that operation several vials of ${ }^{133} \mathrm{Xe}$ were released into the auxiliary blower system, and the response of the detector recorded. This allowed for the quantification of the response, thus calibrating the flow-through system. From these data it was determined that with the sampling system operating at 80 liters per minute, and the blower operating at 350 cfm (single flow rate), the integrated activity in the system is given by the product of the integrated counts times the flow rate in cubic feet per minute times $3 \times 10 "$ millicuries.

Activity (mCi) =

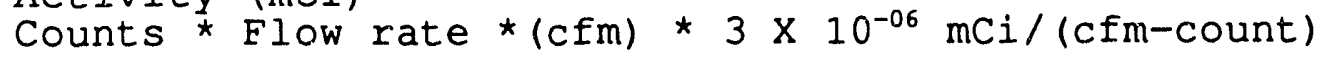

At the standard $350 \mathrm{cfm}$ we have:

Activity (mCi) = Counts $\star 1 \times 10^{-03} \mathrm{mCi} / \mathrm{count}$ 


$$
\begin{array}{r}
\text { DRAFT LANL-NTS-DP-001, R0 } \\
\text { Page } \underline{3} \text { of } \underline{4}
\end{array}
$$

B. Procedure:

Once the presence of chimney gas has been verified, the integrated count of the detector system is calculated from the strip chart. The flow rate in the containment system is determined. The ${ }^{133} \mathrm{Xe}$ activity is then determined using the function given above. Under steady state conditions, the counting rate may be used to calculate the rate of material passing through the system.

The apparent zero time composition of chimney gas has been determined and reported in LA-3420-MS, Analysis of Underground Weapons Test Effluent Samples. The reported composition is:

$$
\begin{array}{ll}
{ }^{131} \mathrm{I} & 1 \\
{ }^{133} \mathrm{I} & 105 \\
{ }^{135} \mathrm{I} & 1360 \\
& \\
{ }^{133} \mathrm{Xe} & 10000 \\
{ }^{135} \mathrm{Xe} & 50000
\end{array}
$$

These data are used to infer the composition of the gas from the observed ${ }^{133} \mathrm{Xe}$ in the mixture. Table 1 is entered with the age of the mixture and the relative amounts of the other constituents determined. These relative amounts are then multiplied by the ${ }^{133} \mathrm{Xe}$ value. 
DRAFT LANL-NTS-DP-001, RO

Page $\underline{4}$ of $\underline{4}$

Table 1

Relative Activity - Chimney Gas

$\begin{array}{cccccc}\begin{array}{c}\text { Age } \\ \text { (DaYs) }\end{array} & 131-\mathrm{I} & 133-\mathrm{I} & 135-\mathrm{I} & 133-\mathrm{Xe} & 135-\mathrm{Xe} \\ 0.0 & 1.00 \mathrm{E}-04 & 1.05 \mathrm{E}-02 & 1.36 \mathrm{E}-01 & 1.00 \mathrm{E}+00 & 5.00 \mathrm{E}+00 \\ 1.0 & 1.05 \mathrm{E}-04 & 5.39 \mathrm{E}-03 & 1.25 \mathrm{E}-02 & 1.00 \mathrm{E}+00 & 9.17 \mathrm{E}-01 \\ 2.0 & 1.10 \mathrm{E}-04 & 2.76 \mathrm{E}-03 & 1.15 \mathrm{E}-03 & 1.00 \mathrm{E}+00 & 1.68 \mathrm{E}-01 \\ 3.0 & 1.15 \mathrm{E}-04 & 1.42 \mathrm{E}-03 & 1.05 \mathrm{E}-04 & 1.00 \mathrm{E}+00 & 3.08 \mathrm{E}-02 \\ 4.0 & 1.20 \mathrm{E}-04 & 7.26 \mathrm{E}-04 & 9.64 \mathrm{E}-06 & 1.00 \mathrm{E}+00 & 5.66 \mathrm{E}-03 \\ 5.0 & 1.26 \mathrm{E}-04 & 3.73 \mathrm{E}-04 & 8.85 \mathrm{E}-07 & 1.00 \mathrm{E}+00 & 1.04 \mathrm{E}-03 \\ 6.0 & 1.32 \mathrm{E}-04 & 1.91 \mathrm{E}-04 & 8.12 \mathrm{E}-08 & 1.00 \mathrm{E}+00 & 1.90 \mathrm{E}-04 \\ 7.0 & 1.38 \mathrm{E}-04 & 9.80 \mathrm{E}-05 & 7.45 \mathrm{E}-09 & 1.00 \mathrm{E}+00 & 3.49 \mathrm{E}-05 \\ 8.0 & 1.45 \mathrm{E}-04 & 5.03 \mathrm{E}-05 & 6.84 \mathrm{E}-10 & 1.00 \mathrm{E}+00 & 6.40 \mathrm{E}-06 \\ 9.0 & 1.52 \mathrm{E}-04 & 2.58 \mathrm{E}-05 & 6.28 \mathrm{E}-11 & 1.00 \mathrm{E}+00 & 1.17 \mathrm{E}-06 \\ 10.0 & 1.59 \mathrm{E}-04 & 1.32 \mathrm{E}-05 & 5.76 \mathrm{E}-12 & 1.00 \mathrm{E}+00 & 2.15 \mathrm{E}-07 \\ 11.0 & 1.66 \mathrm{E}-04 & 6.78 \mathrm{E}-06 & 5.29 \mathrm{E}-13 & 1.00 \mathrm{E}+00 & 3.95 \mathrm{E}-08 \\ 12.0 & 1.74 \mathrm{E}-04 & 3.48 \mathrm{E}-06 & 4.85 \mathrm{E}-14 & 1.00 \mathrm{E}+00 & 7.24 \mathrm{E}-09 \\ 13.0 & 1.82 \mathrm{E}-04 & 1.78 \mathrm{E}-06 & 4.45 \mathrm{E}-15 & 1.00 \mathrm{E}+00 & 1.33 \mathrm{E}-09 \\ 14.0 & 1.91 \mathrm{E}-04 & 9.14 \mathrm{E}-07 & 4.08 \mathrm{E}-16 & 1.00 \mathrm{E}+00 & 2.44 \mathrm{E}-10 \\ 15.0 & 2.00 \mathrm{E}-04 & 4.69 \mathrm{E}-07 & 3.75 \mathrm{E}-17 & 1.00 \mathrm{E}+00 & 4.47 \mathrm{E}-11 \\ 16.0 & 2.10 \mathrm{E}-04 & 2.41 \mathrm{E}-07 & 3.44 \mathrm{E}-18 & 1.00 \mathrm{E}+00 & 8.20 \mathrm{E}-12 \\ 17.0 & 2.19 \mathrm{E}-04 & 1.23 \mathrm{E}-07 & 3.16 \mathrm{E}-19 & 1.00 \mathrm{E}+00 & 1.50 \mathrm{E}-12 \\ 18.0 & 2.30 \mathrm{E}-04 & 6.33 \mathrm{E}-08 & 2.90 \mathrm{E}-20 & 1.00 \mathrm{E}+00 & 2.76 \mathrm{E}-13 \\ 19.0 & 2.41 \mathrm{E}-04 & 3.24 \mathrm{E}-08 & 2.66 \mathrm{E}-21 & 1.00 \mathrm{E}+00 & 5.06 \mathrm{E}-14 \\ 20.0 & 2.52 \mathrm{E}-04 & 1.66 \mathrm{E}-08 & 2.44 \mathrm{E}-22 & 1.00 \mathrm{E}+00 & 9.27 \mathrm{E}-15 \\ 21.0 & 2.64 \mathrm{E}-04 & 8.53 \mathrm{E}-09 & 2.24 \mathrm{E}-23 & 1.00 \mathrm{E}+00 & 1.70 \mathrm{E}-15 \\ 22.0 & 2.77 \mathrm{E}-04 & 4.38 \mathrm{E}-09 & 2.05 \mathrm{E}-24 & 1.00 \mathrm{E}+00 & 3.12 \mathrm{E}-16 \\ 23.0 & 2.90 \mathrm{E}-04 & 2.24 \mathrm{E}-09 & 1.88 \mathrm{E}-25 & 1.00 \mathrm{E}+00 & 5.72 \mathrm{E}-17 \\ 24.0 & 3.03 \mathrm{E}-04 & 1.15 \mathrm{E}-09 & 1.73 \mathrm{E}-26 & 1.00 \mathrm{E}+00 & 1.05 \mathrm{E}-17 \\ 25.0 & 3.18 \mathrm{E}-04 & 5.90 \mathrm{E}-10 & 1.59 \mathrm{E}-27 & 1.00 \mathrm{E}+00 & 1.92 \mathrm{E}-18 \\ 26.0 & 3.33 \mathrm{E}-04 & 3.03 \mathrm{E}-10 & 1.46 \mathrm{E}-28 & 1.00 \mathrm{E}+00 & 3.53 \mathrm{E}-19 \\ 27.0 & 3.49 \mathrm{E}-04 & 1.55 \mathrm{E}-10 & 1.34 \mathrm{E}-29 & 1.00 \mathrm{E}+00 & 6.47 \mathrm{E}-20 \\ 28.0 & 3.65 \mathrm{E}-04 & 7.96 \mathrm{E}-11 & 1.23 \mathrm{E}-30 & 1.00 \mathrm{E}+00 & 1.19 \mathrm{E}-20 \\ 29.0 & 3.82 \mathrm{E}-04 & 4.08 \mathrm{E}-11 & 1.13 \mathrm{E}-31 & 1.00 \mathrm{E}+00 & 2.18 \mathrm{E}-21 \\ 30.0 & 4.00 \mathrm{E}-04 & 2.09 \mathrm{E}-11 & 1.03 \mathrm{E}-32 & 1.00 \mathrm{E}+00 & 3.99 \mathrm{E}-22\end{array}$




\title{
SECTION II.2
}

\section{Operational Area Monitoring Plan}

for the

Lawrence Livermore National Laboratory

Testing Areas and Facilities

Nevada Test Site

\author{
by \\ K. E. Raschke \\ Lawrence Livermore National Laboratory
}

January 21, 1993 


\subsection{Introduction}

\subsection{Organizational Structure and Responsibility}

Effluent monitoring of LLNL programs at the NTS is the responsibility of the LLNL-NTOD ES\&H Group. This group's support to Nuclear Test G.Z. operations is under the direction and control of the LLNL Test Director. All other operations are under the cognizance of the LLNLNTOD Head.

\subsection{Effluent Monitoring Plan}

\subsubsection{Post-Event Monitoring}

During drill-back operations the potential for a release of radioactive materials is greater than the potential for a venting but the amount of radioactivity involved is much smaller. A release through the ventline is the main type of effluent encountered. The ventline is a 20 -inch (outside diameter) steel pipe conducting air from the postshot drilling cellar, through banks of filters, to a point of release to the atmosphere. Also, releases do occur on the rig platform primarily through the top of the drill string. Minor releases of radioactivity may occur from handling of the core material in the core trailer or the "highgrading" (sample preparation) shack. Liquid radioactive drilling fluid effluents are contained (tanks) at the site. 


\title{
SECTION II.3
}

\section{Operational Area Monitoring Plan for the Defense Nuclear Agency/Sandia Testing Areas and Facilities Nevada Test Site}

\author{
by \\ D. A. Bedsun \\ Defense Nuclear Agency \\ J. H. Metcalf \\ Sandia National Laboratory
}

January 21, 1993 


\subsection{Effluent Monitoring Plan}

\subsubsection{Permit Requirements}

Nevada Water Pollution Control Permits are required for the liquid effluent discharged from the tunnels. A temporary water pollution control permit for $N$ Tunnel was issued by the State of Nevada on 15 May 1992. A permanent permit application for this source will be subritted to the State by 11 Sep 1992. Permit applications for $E$ and $T$ Tunnels are being prepared for submittal to the State prior to 31 Dec 1992. There are no permit requirements for occasional low-level releases of airborne radioactive materials. 


\title{
SECTION II.4
}

\section{Operational Area Monitoring Plan for the Liquefied Gaseous Fuels Spill Test Facility Nevada Test Site}

\author{
by \\ H. E. Gray \\ EG\&G Energy Measurements, Inc.
}

January 21, 1993 


\subsection{Effluents}

\subsection{Inventory of Effluents}

Tests may involve any of the approved fluids. These are high vapor pressure materials that become gaseous on release. Test fluids are received at the site several days prior to testing, and any materials not used in tests are returned to the vendor as soon as shipping is arranged.

Table 1-1 List of Approved Chemicals

Ammonia

Bromine

Butane

Carbon Dioxide

Chlorine

Chlorosulfonic Acid

Cyclohexane

Ethylene

Fluorosulfonic Acid

Hydrazine

Hydrogen Sulfide

Hydrogen Fluoride

Hydrogen Chloride

LNG

LPG
Methane

Methyl trichlorosilane

Methylamine

Nitrogen tetroxide

Oleum

Phosgene

Phosphorous oxychloride

Phosphorous trichloride

Propane

Silicon tetrachloride

Sulfur dioxide

Sulfur trioxide

Titanium tetrachloride

Trichlorosilane

Unsymmetrical dimethyl hydrazine 


\section{SECTION II.5A}

\section{Operational Area Monitoring Plan for the Onsite Radiological Surveillance Program Nevada Test Site}

by

F. D. Ferate and S. C. Black

Reynolds Electrical \& Engineering Co., Inc.

January 21, 1993 


\subsection{Effluents (Emissions and Discharges)}

\subsubsection{Radioactive Liquid Discharges}

Liquid discharges (process water) from the TTF will be decontaminated to meet Safe Drinking Water Act (SDWA) limits and discharged into existing sewage lagoons or, if SDWA limits cannot be met, stored until it may be released to permitted containment ponds located elsewhere on the NTS.

\subsection{Effluent Monitoring Plan}

\subsubsection{Liquid Discharges}

Liquid radioactive waste from Area 12 tunnels and the Area 6 Decontamination Facility discharges into holding ponds; radioactive liquid discharges from the TTF will be decontaminated to levels below SDWA release limits and discharged as sanitary waste into existing sewage treatment facilities. Any TTF liquid effluent exceeding SDWA radioactivity limits will be stored in tanks until it may be discharged into permitted containment ponds located elsewhere on the NTS.

\subsubsection{Area 25 Treatablilty Test Facility}

The REECo Treatability Test Facility laboratory located at Area 25 in Building 3124 tests various physical separations technologies for removing low concentrations of americium, plutonium, and uranium species from native soils. Concentrations of these species shall never exceed $100 \mathrm{nCi} / \mathrm{g}$ and will average approximately $500 \mathrm{pCi} / \mathrm{g}$.

Processing likely to produce nuisance dust will be performed wet and in containments. All laboratory test bay and laboratory hood exhausts will be vented to the outside through HEPA filters. The potential for release of radioactivity to the environment is directly proportional to the laboratory sample throughput. Maximum annual sample throughput will not exceed 60 tons.

Compliance with NESHAP monitoring criteria requires a determination of the type of effluent monitoring that is necessary. The activity levels of radionuclides that may be released from this facility are such that doses to offsite individuals are expected to be $<<0.1 \mathrm{mrem}$, therefore, only periodic confirmatory measurements are required followed by calculating dose $\left(\mathrm{H}_{\mathrm{E})}\right.$ for normal operations and assuming that all emission controls are inoperative.

Assuming a worst case release to the environment of the maximum annual 60 ton throughput, the average concentration of $500 \mathrm{pCi} / \mathrm{g}$, and a respirable factor of 0.5 , the maximum individual annual exposure to a member of the general public may be calculated. Using EPA's CAP88PC computer model, the Desert Rock Star, along with assuming that the ${ }^{238} U$ was separated from its daughter isotopes and all consumption was from locally produced food, an annual dose of $<10^{-4}$ mrem is calculated for the nearest resident (Lathrop Wells). This is negligibly small when compared to the NESHAP threshold of $0.1 \mathrm{mrem}$ and limit of $10 \mathrm{mrem}$. 


\subsection{Analytical Procedures}

\subsection{Analyses Employed}

Uranium analyses are required because the Treatability Test Facility is responsible for studying physical methods of removal of uranium contamination from soils consequently quantitative uranium analysis is required for determination of removal efficiencies. 


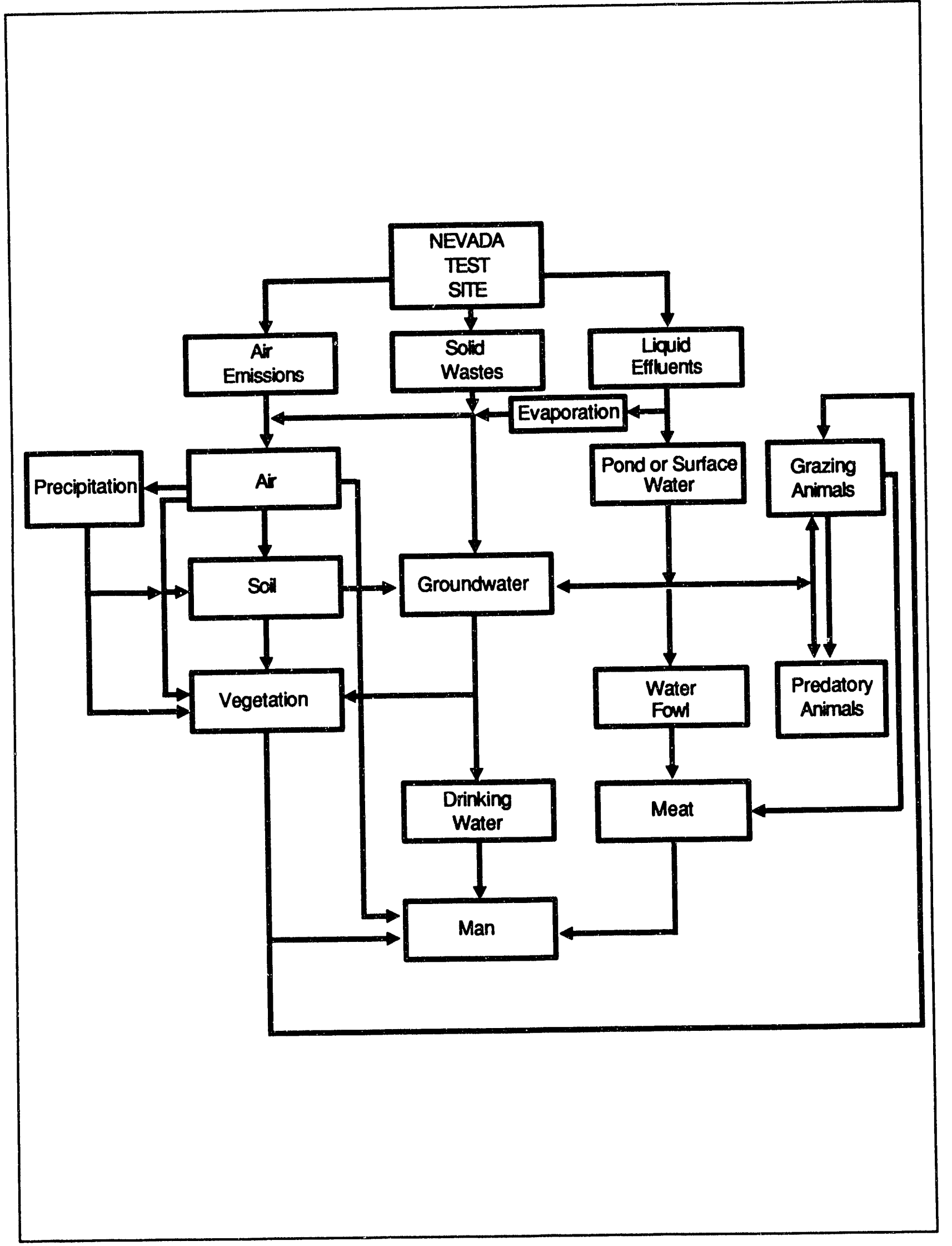

Exhibit Potential Exposure Pathways Diagram 


\begin{tabular}{|c|c|c|c|c|}
\hline SAMPLE TYPE & DESCRIPTION & $\begin{array}{l}\text { COLLECTION } \\
\text { FREQUENCY }\end{array}$ & $\begin{array}{l}\text { NUMBER OF } \\
\text { SAMPLING } \\
\text { LOCATIONS }\end{array}$ & $\begin{array}{l}\text { TYPE OF } \\
\text { ANALYSIS }\end{array}$ \\
\hline \multirow[t]{3}{*}{ Air } & $\begin{array}{l}\text { Continuous sampling } \\
\text { through Whatman } \\
\text { GF/A glass filter and } \\
\text { a charcoal cartridge }\end{array}$ & Weokly & 52 & $\begin{array}{l}\text { Gamma } \\
\text { Spectroscopy, } \\
\text { gross beta, }{ }^{230} \mathrm{Pu} \\
\text { (monthly } \\
\text { composite) }\end{array}$ \\
\hline & $\begin{array}{l}\text { Low-volume sampling } \\
\text { through silica gel }\end{array}$ & Biweokly & 17 & HTO (tritium) \\
\hline & $\begin{array}{l}\text { Continuous, low- } \\
\text { volume sampling }\end{array}$ & Woakly & 10 & ${ }^{2} \mathrm{~K}_{\mathrm{r}}$ and $133 \mathrm{x}_{0}$ \\
\hline Potable Water & 1-liter grab sample & Woekly & 9 & $\begin{array}{l}\text { Gross } \alpha, \text { Gamma* } \\
\text { Spectroscopy, } \\
\text { gross B, tritium, } \\
{ }^{230} \mathrm{Pu} \text { (quarterly) }\end{array}$ \\
\hline Supply Wells & 1-liter grab sample & Monthly & 12 & $\begin{array}{l}\text { Gross } \alpha, \text { Gamma* } \\
\text { Spectroscopy, } \\
\text { gross B, tritium, } \\
{ }^{230} \mathrm{Pu} \text { (quarterly) }\end{array}$ \\
\hline Open Reservoirs & 1-liter grab sample & Monthly & 16 & $\begin{array}{l}\text { Gamma } \\
\text { Spectroscopy, } \\
\text { gross B, tritium, } \\
{ }^{230} \text { Pu (quarterly) }\end{array}$ \\
\hline Natural Springs & 1-liter grab sample & Monthly & 7 & $\begin{array}{l}\text { Gamma } \\
\text { Spectroscopy, } \\
\text { gross B, tritium, } \\
{ }^{230} \mathrm{Pu} \text { (quarterly) }\end{array}$ \\
\hline Containment Ponds & 1-liter grab sample & Monthly & 9 & $\begin{array}{l}\text { Gamma } \\
\text { Spectroscopy, } \\
\text { gross B, tritium, } \\
\text { 230pu (quarterty) }\end{array}$ \\
\hline Sewage Ponds & 3-liter grab sample & Quarterly & 3 & $\begin{array}{l}\text { Gamma } \\
\text { Spectroscopy, } \\
\text { gross B, tritium, } \\
{ }^{230} \mathrm{Pu}\end{array}$ \\
\hline $\begin{array}{l}\text { External Gamma } \\
\text { Radiation Levels }\end{array}$ & $\begin{array}{l}\text { UD-814AS } \\
\text { Thermoluminescent } \\
\text { Dosimeters }\end{array}$ & Quarterly & 187 & $\begin{array}{l}\text { Total integrated } \\
\text { exposure over } \\
\text { field cycle }\end{array}$ \\
\hline \multicolumn{5}{|c|}{$\begin{array}{l}\text { - }{ }^{228,228} \mathrm{Ra}, \mathrm{Rn} \text { and } U \text { analysis of potable supply wells and water if gross } \alpha>5 \text { pCir. Samples are } \\
\text { collected annually from each water source for }{ }^{\circ} \mathrm{Sr} \text { analysis }\end{array}$} \\
\hline
\end{tabular}

Exhibit 4-6 Summary of the NTS Radiological Surveillance Program 


\begin{tabular}{|c|c|c|c|c|c|c|}
\hline $\begin{array}{l}\text { TYPE OF } \\
\text { ANALYSIS }\end{array}$ & $\begin{array}{l}\text { TYPE OF } \\
\text { SAMPLE }\end{array}$ & $\begin{array}{l}\text { ANALYTTICAL } \\
\text { EQUIPMENT }\end{array}$ & $\begin{array}{l}\text { COUNTING } \\
\text { TIME(MIN) }\end{array}$ & $\begin{array}{l}\text { ANALYTICAL } \\
\text { PROCEDURES }\end{array}$ & $\begin{array}{l}\text { SAMPLE } \\
\text { SIZE }\end{array}$ & 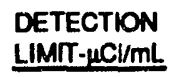 \\
\hline$m_{s r}$ & Water & $\begin{array}{l}\text { Gas-Flow } \\
\text { Proportional } \\
\text { Counter }\end{array}$ & 100 & $\begin{array}{l}\text { Precipitate hydroxides, } \\
\text { scetates, finally carbonate. } \\
\text { Calculate by yttium } \\
\text { ingrowth. }\end{array}$ & $1000 \mathrm{~mL}$ & $2 \times 10^{\circ}$ \\
\hline$m_{m a}$ & Water & $\begin{array}{l}\text { Germanium } \\
\text { Sermiconductor }\end{array}$ & 500 & $\begin{array}{l}\text { Ra tracer, precipitate with } \\
\text { barium sulphate, gamma } \\
\text { count. }\end{array}$ & $1000 \mathrm{~mL}$ & $2 \times 10^{\circ}$ \\
\hline Uranium & Water & $\begin{array}{l}\text { Sillicon } \\
\text { Semiconductor }\end{array}$ & 1000 & $\begin{array}{l}\text { Separate on ion exchange, } \\
\text { plate on stainless planchet }\end{array}$ & $1000 \mathrm{~mL}$ & $8 \times 10^{\prime \prime 1}$ \\
\hline \multirow[t]{2}{*}{ Gross Beta } & Air & $\begin{array}{l}\text { Gas-fiow } \\
\text { Proportional } \\
\text { Counter }\end{array}$ & 20 & $\begin{array}{l}\text { Place fitter on a } 12.7 \mathrm{~cm} \\
\text { stainless steel planchet }\end{array}$ & $10^{\circ} \mathrm{mL}$ & $2 \times 10^{-10}$ \\
\hline & Water & $\begin{array}{l}\text { Gas-flow } \\
\text { Proportional } \\
\text { Counter }\end{array}$ & 100 & $\begin{array}{l}\text { Evaporate, transfor residue } \\
\text { to a } 12.7 \mathrm{~cm} \text { stainless stoel } \\
\text { planchet }\end{array}$ & $1000 \mathrm{~mL}$ & $1 \times 10^{\circ}$ \\
\hline \multirow[t]{3}{*}{$\begin{array}{l}\text { Gamma } \\
\text { Spectroscopy }\end{array}$} & $\begin{array}{l}\text { Air } \\
\text { (particulate) }\end{array}$ & $\begin{array}{l}\text { Germanium } \\
\text { Semiconductor }\end{array}$ & 20 & $\begin{array}{l}\text { As for gross bota, but in } \\
\text { plastic bag }\end{array}$ & $10^{\circ} \mathrm{mL}$ & $5 \times 10^{-18}$ \\
\hline & $\begin{array}{l}\text { Air } \\
\text { (gaseous) }\end{array}$ & $\begin{array}{l}\text { Germanium } \\
\text { Semiconductor }\end{array}$ & 20 & $\begin{array}{l}\text { Place charcosal cartridge in } \\
\text { plastic bag }\end{array}$ & $10^{\circ} \mathrm{mL}$ & $5 \times 10^{-16}$ \\
\hline & Water & $\begin{array}{l}\text { Germanium } \\
\text { Semiconductor }\end{array}$ & 20 & $\begin{array}{l}\text { Aliquot sample into Nagene } \\
\text { bottie }\end{array}$ & $500 \mathrm{~mL}$ & $1 \times 10^{-1}$ \\
\hline$\Delta \mathrm{K}_{\mathrm{r}}$ & Air & $\begin{array}{l}\text { Liquid } \\
\text { Scintillation } \\
\text { Counter }\end{array}$ & 300 & $\begin{array}{l}\text { Cryogenic-gus chro- } \\
\text { matographic techniques } \\
\text { used to collect krypton into } \\
\text { liquid scintillation solution }\end{array}$ & $3 \times 10^{5} \mathrm{~mL}$ & $25 \times 10^{-12}$ \\
\hline \multirow[t]{2}{*}{$m e$} & Air & $\begin{array}{l}\text { Silicon } \\
\text { Semiconductior }\end{array}$ & 333 & $\begin{array}{l}\text { Filter is ashed and put in } \\
\text { solution. Pu is purified by } \\
\text { anion exchange resin } \\
\text { column, then } \\
\text { electrodeposited on a } \\
\text { stainless stoel disc }\end{array}$ & $4 \times 10^{\circ} \mathrm{mL}$ & $1 \times 10^{-17}$ \\
\hline & Water & $\begin{array}{l}\text { Silicon } \\
\text { Semiconductor }\end{array}$ & 1000 & $\begin{array}{l}\text { Pu is concentrated with } \\
\text { Fo(OH)3 and purified with } \\
\text { anion resin column. } \\
\text { Electrodeposited on a } \\
\text { stainless steol disc }\end{array}$ & $1000 \mathrm{~mL}$ & $4 \times 10^{.11}$ \\
\hline \multirow[t]{2}{*}{ Tritium } & Air & $\begin{array}{l}\text { Liquid } \\
\text { Scintillation } \\
\text { Counter }\end{array}$ & 70 & $\begin{array}{l}\text { Distill the } \mathrm{H}_{2} \mathrm{O} \text { and aliquot } 5 \\
\mathrm{~mL} \text { into \& scintillation } \\
\text { solution }\end{array}$ & $1 \times 10^{7} \mathrm{~mL}$ & $3 \times 10^{.13}$ \\
\hline & Water & $\begin{array}{l}\text { Liquid } \\
\text { Scinillation } \\
\text { Counter }\end{array}$ & 70 & $\begin{array}{l}\text { Distill } 20 \mathrm{~mL} \text { of Sample, } 5 \\
\mathrm{~mL} \text { aliquot into scintillation } \\
\text { solution }\end{array}$ & $5 \mathrm{~mL}$ & $4 \times 10^{-7}$ \\
\hline${ }^{2} x_{\theta}$ & Air & $\begin{array}{l}\text { Liquid } \\
\text { Santillation } \\
\text { Countor }\end{array}$ & 300 & $\begin{array}{l}\text { Cryogenic-gas } \\
\text { chromatographic tochniques } \\
\text { used to collect xenon into } \\
\text { liquid scintillation solution }\end{array}$ & $3 \times 10^{5} \mathrm{~mL}$ & $25 \times 10^{-12}$ \\
\hline $\begin{array}{l}\text { Direct Gamma } \\
\text { Radiation }\end{array}$ & TLD & $\begin{array}{l}\text { Panasonic UD } \\
710 A \text { TLD } \\
\text { Reader }\end{array}$ & & Automated & & $10 \mathrm{mR}$ quarter \\
\hline
\end{tabular}

\section{Exhibit 5-1 Summary of Laboratory Analytical Procedures}




\title{
SECTION II.5B
}

\section{Operational Area Monitoring Plan for Nonradiological Monitoring at the Tonopah Test Range and Nevada Test Site}

\author{
by \\ S. A. Wade and F. D. Ferate \\ Environmental Compliance Office \\ and the Environmental Section \\ Reynolds Electrical \& Engineering Co., Inc.
}

January 21, 1993 


\subsection{Introduction}

This Section documents Reynolds Electrical \& Engineering Company, Inc.'s (REECo's) nonradiological effluent and environmental monitoring services for the Nevada Test Site (NTS) and the Tonopah Test Range (TTR) provided under contract with DOE/NV. Environmental monitoring including ecological monitoring, permit application processing, and reporting the compliance status of user organizations at the NTS and TTR have also been assigned to REECo.

\subsection{Operational Activities}

- Site-wide National Environmental Policy Act (NEPA) compliance in areas involving

- Construction, relocation, expansion, removal, or modification of facilities and/or test sites on the NTS

- Disposal of hazardous wastes

- Monitoring of flora and fauna in selected areas of the NTS to detect and investigate ecological changes due to DOE activities or natural causes.

\subsection{Rationale}

The purpose of the REECo environmental surveillance program for nonradiological substances is to examine, measure, document, and compare environmental contaminant levels with laws, regulations, standards, and/or permit requirements. The CAA mandates that asbestos removal and renovation projects be monitored and the results reported to the EPA under NESHAP requirements. The primary environmental permit areas for the NTS involve air quality and RCRA requirements. A National Pollution Discharge Elimination System (NPDES) permit is being prepared for the NTS tunnel discharge systems. In addition, an EPA Form 1 Application will be submitted for both NTS and TTR as the first step in NPDES permitting. A General Permit for industrial Source storm water runoff will be developed pending final ruling by EPA.

The purpose of the ecological monitoring on the NTS (performed in the past by REECo and UCLA; assigned solely to REECo starting in 1993) is to characterize biological changes caused by DOE activities, measure trends in biological populations, identify and monitor causes of biological problems, and to notify DOE of any biological problems discovered. DOE Order 5400.1 requires that environmental surveillance be conducted to monitor the effects, if any, of DOE activities on onsite and offsite environmental and natural resources. NEPA and Executive Order 11514 require federal agencies to monitor, evaluate and control their agencies' activities so as to protect and enhance the quality of the environment.

\subsection{Liquid Discharges}

\subsubsection{Domestic Sewage}

Sewage discharge permits have been issued by the state of Nevada for discharges to lagoons in Areas $2,6,12,22,23$, and 25 of the NTS and the sewage treatment plant on the TTR. There are no effluent discharges from the lagoons. A variance was granted in 1990 by the 
Nevada Department of Human Resources, Health Division, for the Area 11 Tweezer Facility septic tank and evapotranspiration bed. Substandard percolation rates indicated that a septic tank with an evapotranspiration bed would be more appropriate than a septic tank with field drain system. The septic tank and evapotranspiration bed were installed in September 1990. The sewage lagoon discharge permit for the TTR was reapplied for in July 1992 with an associated request that the permit be transferred to the U.S. Air Force for management and oversight. As of September 1992, the renewed permit for the TTR lagoon system has not been returned by the state of Nevada. The active sewage discharge permits are listed in Table 2-1. This table reflects the status of sewage discharge permits as of August 31, 1992.

Table 2-1 NTS and TTR Sewage Discharge Permits, 1992

\begin{tabular}{lcc} 
Permit No./Area & Date Issued & Expiration Date \\
\cline { 2 - 3 } NEV87069/2\&6 & $02 / 28 / 89$ & $02 / 28 / 94$ \\
NEV87076/22\&23 & $02 / 28 / 89$ & $02 / 28 / 94$ \\
NEV87060/25:6 & $03 / 31 / 88$ & $03 / 31 / 93$ \\
NEV87059/12 & $02 / 28 / 89$ & $02 / 28 / 94$ \\
NEV20001/TTR & $09 / 19 / 87$ & $08 / 20 / 92^{(1)}$ \\
(1) A permit renewal package was transmittod to the state in July, 1992.)
\end{tabular}

\subsubsection{Injection Wells}

Following cessation of discharge, a discharge permit application was retracted by DOE following submission to the state of Nevada for two shallow injection wells at the Area 1 Subdock. The wells were sealed in February 1990. A closure plan for these wells is currently under review by the state. The original steam cleaning operation was replaced with a closed loop recirculating system in May 1991.

\subsection{Air Emissions}

\subsubsection{Permits}

Over the past year, new air quality permits were issued at the NTS for destemming equipment, a portable crusher and screens, and the two-part epoxy batch plant. The state is currently evaluating four permit applications for the portable and stationary mud plants, a process description for the sand bag facility, and a list of approximately $\mathbf{5 0}$ pieces of equipment housing internal combustion engines.

\subsection{Miscellaneous Sampling}

Miscellaneous sampling and analyses for nonradiological substances is performed to confirm or deny the presence of RCRA hazardous wastes. Additionally, sampling and analysis is performed on NTS potable drinking water systems for compliance with primary and secondary 
standards of the Safe Drinking Water Act. Finally, sampling and analysis for biological oxygen demand, total suspended solids, and dissolved oxygen is performed on certain NTS sewage lagoon systems as required under the discharge permit (see section 3.1.3.1).

\subsection{Ecological Monitoring}

The ecological monitoring effort on the NTS includes monitoring of undisturbed control plots in the ecosystems impacted by nuclear tests to determine natural baseline conditions, monitoring of study plots in representative disturbed areas to determine the impact of the disturbance and investigate and document natural recovery processes, and monitoring of larger wildlife throughout the NTS to estimate populations and distributions and track changes in these with time. Undisturbed control plots and disturbed plots are surveyed at different frequencies for perennial and ephemeral plants, and reptiles (lizards) and small mammals. Counts are made of wild horses, and field observations and worker reports on raptors, waterfowl, lions, deer and ravens furnish knowledge of approximate densities and ranges of these species. Detailed records have been kept for many years of the desert tortoise population in the Rock Valley study enclosure on the NTS.

\subsection{Effluents (Emissions and Discharges)}

\subsection{Monitoring System Design - Water and Wastewater}

\subsubsection{Drinking Water}

To support the diverse work areas at the NTS which are not supplied by a water well and distribution system, poiable water is hauled from a fill stands located in Areas 6, 12, and 23. Each load of water is chlorinated and sampled for analysis by the APL. If the sample from a water truck load exceeds the state regulatory requirement for coliform bacteria, both the system or tank to which the water was hauled as well as the truck are removed from service. The truck is superchlorinated and resampled according to state requirements. In order to return the truck to service, samples collected for four consecutive days must have a coliform count that is below state regulatory requirements. A total of 1134 water truck samples were collected during 1991.

\subsection{Monitoring System Design - Air Emissions}

\subsubsection{Air Emissions}

Air emissions were the focus of an air quality study conducted by Engineering-Science, Pasadena, California, at the NTS during August and September 1990, and a subsequent study performed by the Mark Group in March through June 1992. These monitoring programs were conducted under subcontract to REECo to determine the compliance status of the NTS with current air quality standards specified by state and federal regulations.

The emissions from four point sources were tested by Engineering-Science to assess compliance with permit conditions and applicable state and federal standards. Among the sources tested were a boiler, paint spray booth, incinerator, and tunnel exhaust. These sources are representative of equipment operated at the NTS which have the potential to emit 
regulated airborne pollutants. This study concluded that the emissions from these sources were within permit and state and federal standards.

The Mark Group study objectives were to: (1) identify specific sources of particulate emissions at designated facilities and operations within the NTS; (2) determine BACT to reduce particulate emissions at the specified facilities and operations; (3) provide estimated costs for the BACT; and (4) compile a list of manufacturers and vendors of dust control equipment.

Costs to retrofit equipment were compared with the costs of purchasing new equipment. Recommendations made by the Mark Group included the installation of electrostatic precipitators at the Area 1 Shaker Plant and the Area 12 P-tunnel vent; a cyclone collection system for both the Area 1 Batch Plant and the Portec Hopper (currently in Area 3, tentatively planned to be moved to the Area 1 Batch Plant) and for the Area 1 Rotary Dryer; provide tent enclosures for the Area 1 Crusher, Areas 2 and 3 stemming equipment and the Area 3 Twopart Epoxy Batch Plant. Dust from heavily-used unpaved roads may be mitigated through paving, water, or chemical surfactants. The above recommendations, along with less expensive alternatives, are under consideration.

The Mark Group study confirmed the exceedance of state requirements for particilate emissions for major NTS emission sources. Since particulate emissions providt the greatest concern for air quality environmental surveillance, REECo Environmental Compli ince Office (ECO) personnel routinely perform scheduled and unscheduled VE surveillances of equipment and facilities which have air quality operating permits to verify compliance with particulate opacity limitations. Where VE surveillance identifies an exceedance of permit opacity limitations, corrective action is undertaken. As discussed in section 3.2.1, the recommendations from the Mark Group study are aiso under consideration.

\subsection{Environmental Surveillance}

\subsection{Standard Operating Procedures/Implementing Procedures}

The following listing of procedures states the type of sampling and the method of collecting those samples. Environmental surveillance, effluent monitoring and ecological monitoring procedures are included.

Ecological Monitoring

AABAF.D.02.10

AABAF.D.03.10

AABAF.D.04.00

AABAF.D.05.00

AABAF.D.06.00

AABAF.D.07.00
Perennial Plant Sampling Procedures

Ephemeral Plant Sampling Procedures

Tortoise Detailed Procedure

Lizard Study Procedures

Small Mammal Study Procedures

Procedure for Studying Individual Plants and Animals 


\section{REFERENCES}

U.S. Department of Energy Nevada Operations Office Annual Site Environmental Report 1991, Reynolds Electrical \& Engineering Co., Inc., Report Number DOE/NV/10630-33. 


\title{
SECTION II.5C
}

\section{Operational Area Monitoring Plan for the Waste Management Department Nevada Test Site}

\author{
by \\ M. E. Donahue \\ Reynolds Electrical \& Engineering Co., Inc.
}

January 21, 1993 


\subsection{Effluents (Emissions and Discharges)}

The RWMS pits in Area 5 may be considered to be diffuse effluent sources. A diffuse source is an area source or several point sources near each other. The disposal site, along with other NTS sites, will be investigated and assessed according to the requirements of DOE Order 5400.1 to determine whether or not they will be classified as effluent sources.

\subsection{Environmental Surveillance Plan}

\section{4. $\quad$ Meteorological Monitoring}

A meteorological station is on order for the RWMS. The station will collect continuous wind speed and direction measurements and precipitation, temperature, relative humidity, solar heat flux, and soil heat flux data.

Table 4-1 summarizes the environmental surveillance collection and analysis activities for the Area 5 RWMS and the Area 3 BWMF.

\subsection{Ongoing Waste Management Studies}

Results from studies of site characterization and vadose zone monitoring, $w$ be evaluated as they becorine available and may necessitate alteration of sampling locations and/or frequencies.

\subsection{Surveillance Description}

\subsubsection{Soil}

Soil samples are taken on grids to minimize the bias from uneven distributions of radioactive materials which may have migrated to the surface. Samples may also be collected from locations susf ected of contamination.

Soil samples are taken to detect and determine the extent of migration of disposed materials to the surface. Each sample collected will be analyzed by gamma spectrcscopy, tritiuin, grcis alpha and beta. Twenty-five percent of the samples will be analyzed for ${ }^{91} \mathrm{Sr},{ }^{241} \mathrm{Am},{ }^{238+239} \mathrm{Pu}$ and ${ }^{238} \mathrm{U}$. Samples will be collected at selected points every two years.

\subsubsection{Vadose Zone}

The vadose zone monitoring system is under development. The monitoring system will provide an early detection of migration of hazardous material and LLW into ing vadose zone. 


\subsection{Public Dose Assessment}

According to DOE/EH-0173T, Chapter VIII, estimates of the offsite dose to affected members of the public shall be made using a computer model which is in compliance with 40 CFR 61 , "National Emission Standards for Hazardous Air Pollutants" (NESHAP). Emission monitoring and compliance procedures for DOE facilities require the use of CAP-88 or AIRDOS-PC computer models or other approved procedures to calculate effective dose equivalents to members of the public exposed to airborne radioactivity originating in DOE facilities.

The NESHAPS report is prepared annually by REECo to comply with 40CFR61. The report includes the calculated source term and dose assessment to the maximaily exposed individual residing in any offsite location. 


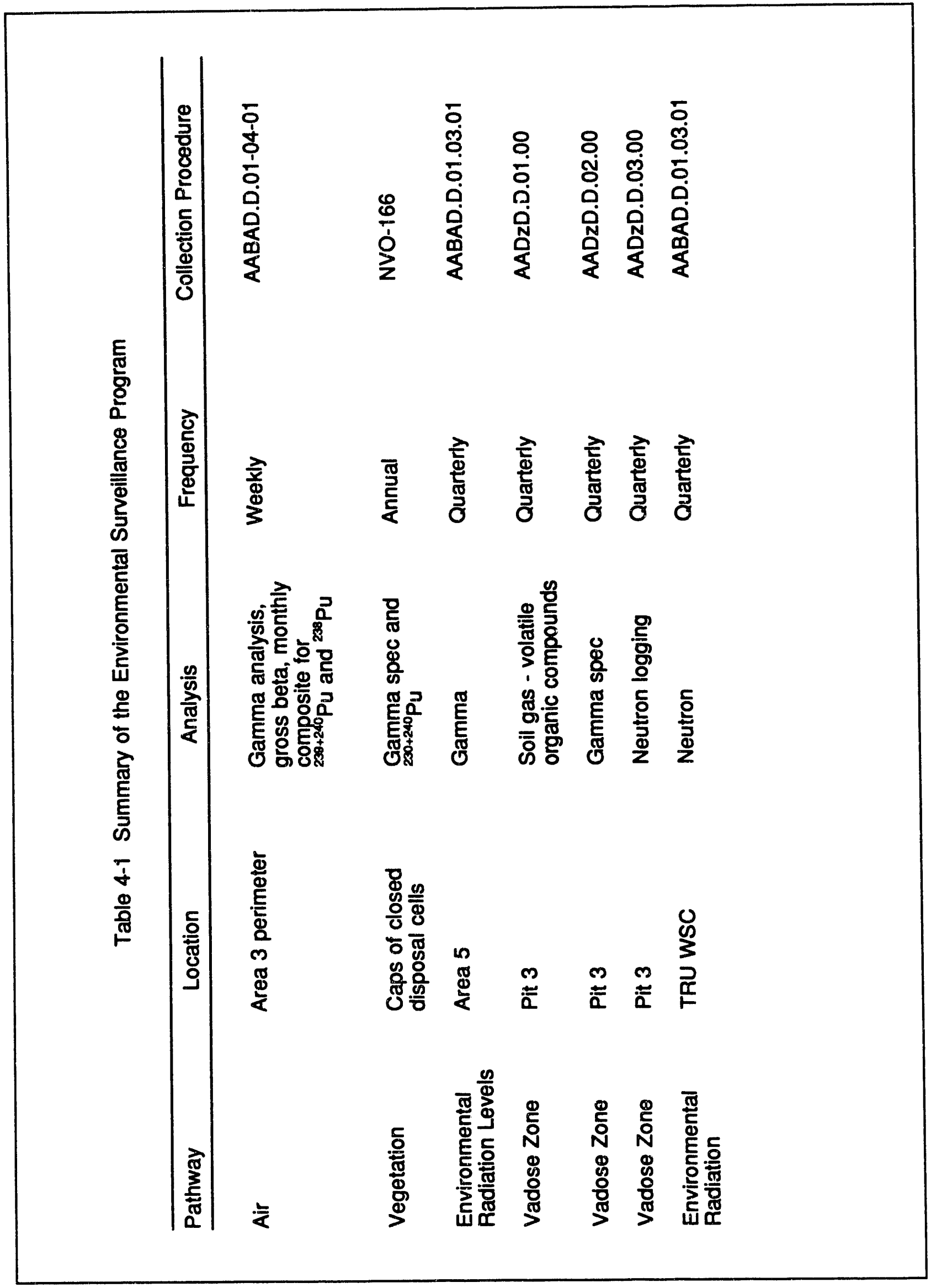




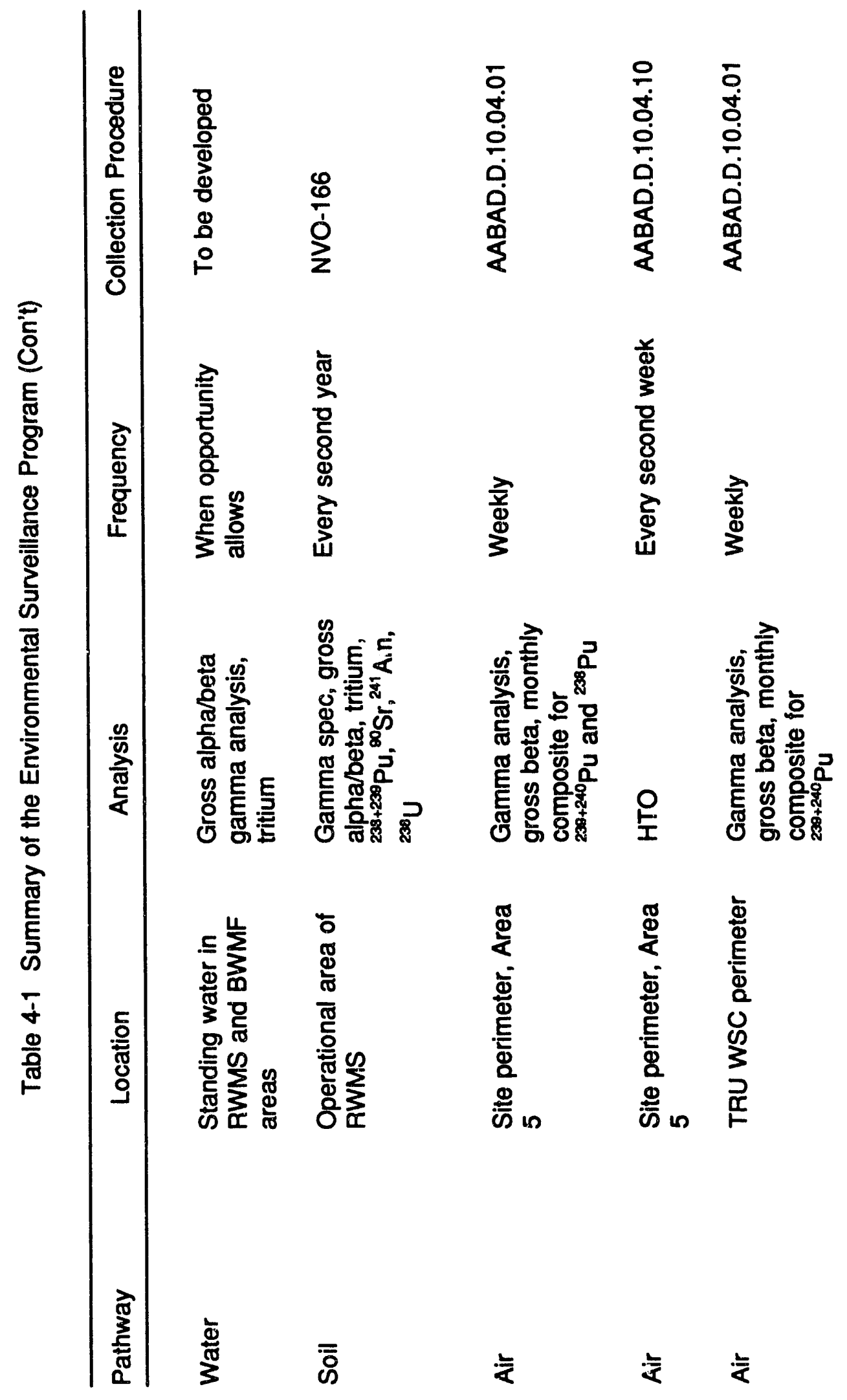




\section{SECTION II.6}

\section{Operational Area Monitoring Plan for Test Support Services Nevada Test Site}

A. E. Smith

Environmental Operations Department

Raytheon Services Nevada

January 21, 1993 


\subsection{Introduction}

One DOE/NV support facility in Las Vegas generates effluents with the potential for environmental contamination. This is:

- Technical Information Production and Services Facility located at 3281 South Highland Drive, Suite 810.

This facility conducts film processing activities which result in the generation of spent photographic processing solutions. These solutions are discharged through the Clark County sewer system following removal of the hazardous constituent (silver) and the adjustment of pH.

\subsection{Effluents (Emissions and Discharges)}

\section{$2.1 \quad$ NTS Facilities}

\subsubsection{Inventory of Surface Water Discharges}

Steam cleaning of trucks in the Area 6, Well 3 Yard has resulted in the discharge of liquid effluents containing oil and grease to the wash down bay floor sump which drains to an unlined pit. Because RSN views this as a direct violation of environmental regulations, the practice has been discontinued. Engineering drawings have been completed for a new outdoor wash down pad which will include a closed loop waste recirculation system with an oil/water separator. The sump is planned to be plumbed into this system, at which time steam cleaning may be resumed. No effluent will result from the closed loop system.

In the interim, a temporary permission may be obtained from the state of Nevada allowing discharge to the pit. Should this "occur, the effluent monitoring as discussed in Sections 3.1 .2 and 3.2.2 would be applied to these steam cleaning activities.

\section{$2.2 \quad$ NV Support facilities}

\subsubsection{Inventory of Liquid Discharges}

The only discharge is the spent photographic fixer solution containing silver from the Technical Information Products and Services Facility as identified in Section 1.0.

\subsection{Effluent Monitoring Plan}

\subsection{Effluent Monitoring Design Criteria}

\subsubsection{Spent Photographic Solutions from Las Vegas Facilities}

Discharge of spent photographic solutions from the Technical Information Products and Services Facility is constrained by Clark County Permit No. CCSD 0024. Silver discharge to 
to the sewer system is limited to concentrations of $5 \mathrm{mg} / \mathrm{L}$ ( 5 parts per million) or less . In addition, the solutions must exhibit a pH in the range of 4.5 to 9.0 .

Data quality objectives are designed to meet the discharge criteria for concentration and $\mathrm{pH}$. In order to assure compliance with the conditions of the discharge permit, all spent fixer is collected following use. Fixer is processed for silver recovery in accordance with Technical Information Products and Services Operating Procedure 001. Treated solutions are analyzed by the Lockheed Analytical Laboratory in Las Vegas. In addition, each processed effluent is tested with a specific ion probe indicating the presence of silver. Until a sufficient data base is developed to demonstrate the adequacy of the specific ion probe technique, laboratory results will be required prior to disposal to the sewer system. This requirement also pertains to any changes in the process or following system failure. The permit requires semiannual sampling and reporting to Clark County. Laboratory results will be the basis for this report even if the ion probe technique is adequate.

\subsection{Quality Assurance and Quality Control}

The quality assurance and quality control of environmental and effluent data will be in accordance with the Management Quality Assurance Program. The RSN Quality Assurance Division and the RSN Environmental, Safety and Health Compliance Department will routinely audit the environmental effluent data, calculations, and reports prepared by and for the RSN Environmental Operations Department. 


\title{
Operational Area Monitoring Plan For the Offsite Radiological Safety Program Nevada Test Site
}

\author{
by \\ S. C. Black \\ Analytical Services Department \\ Reynolds Electrical \& Engineering Co., Inc. \\ and \\ D. J. Chaloud and A.C. Neale \\ Nuclear Radiation Assessment Division \\ Environmental Monitoring Systems Laboratory \\ U.S. Environmental Protection Agency
}

January 21, 1993 


\subsection{Environmental Surveillance Plan}

\subsection{Rationale}

There are several reference levels for radiation exposure, specified by the International Commission on Radiological Protection (ICRP), DOE, and EPA, to be observed when establishing a monitoring program. Guidelines are written such that all pathways that lead to the exposures shall be routinely monitored if there is a potential for one of the following situations:

- A 1 mrem annual effective dose equivalent to any offsite individual

- A 100 person-rem annual collective effective dose equivalent per million individuals within $80 \mathrm{~km}$ (50 mi) of the site center

- A 5 mrem annual whole-body dose equivalent or 15 mrem to the skin of offsite individuals

- Any exposure to an offsite person of 25 mrem effective dose equivalent in any year (required to be reported to DOE Headquarters)

- Unplanned releases of radioactivity shall be monitored and quantified

All of the above shall be based on statistically significant differences between the point of measurement and the average background in the area, or other suitable control data (DOE, 1991).

The standards for exposure from routine operations, from which the above criteria were derived, are as follows:

- Air Emissions - $10 \mathrm{mrem} / \mathrm{yr}$ (0.1 mSv/yr) to any offsite person (40CFR61)

- Drinking Water - $4 \mathrm{mrem} / \mathrm{yr}(0.04 \mathrm{mSv} / \mathrm{yr})$ based on drinking 2 Lday (40CFR141)

- Sum of all pathways - $100 \mathrm{mrem} / \mathrm{yr}(1 \mathrm{mSv} / \mathrm{yr})$ set forth in ICRP-39

In case of emergency situations:

- The Food and Drug Administration (FDA) PAG is 1500 mrem (15 mSv) to the infant thyroid or 500 mrem (5 mSv) to the whole body, bone marrow, or any other organ from ingestion of food (FDA, 1982).

- Recently revised EPA PAGs recommend evacuation at a projected dose of 1 to 5 rem in the early phase of a nuclear incident (EPA, 1992). Sheltering is an alternative to evacuation under certain specified conditions (EPA, 1992).

- Another useful guide as recommended by the ICRP is that $500 \mathrm{mrem}(5 \mathrm{mSv}$ ) committed effective dose equivalent in a year is acceptable as long as the average over 70 years does not exceed $100 \mathrm{mrem} / \mathrm{yr}$ (1 mSv/yr) (ICRP-39). 


\subsubsection{Radiochemistry}

At concentrations greater than 10 times the MDC, precision is required to be within $\pm 10 \%$ for conventional tritium analyses, uranium, thorium (all media), and strontium (except in milk) and within $\pm 20 \%$ for enriched tritium analyses, strontium (in milk), noble gases, and plutonium. Accuracy objectives for concentrations greater than 10 times the MDC are no greater than \pm $10 \%$ bias for conventional tritium analyses, plutonium, uranium, and thorium and no greater than $\pm 20 \%$ bias for noble gases, enriched tritium, and strontium. At concentrations less than 10 times the MDC, both precision and accuracy are not to exceed \pm 30 percent for all analyses and all media types.

\subsection{Network Design}

\subsubsection{Sample Collection Locations}

The present network locations are primarily the result of historical instrument placement. A statistically based design was not used, nor were any citing criteria established, except that monitoring is done at all inhabited locations within $160 \mathrm{~km}$ (100 mi.) of the NTS, if possible. Availability of electric power is the primary restriction in station location, with the exception of PICs, which can operate on power produced by solar panels. At long distances from Las Vegas, station location is further restricted by the availability of an individual to service the station. Year-round station access is required, which restricts placement of stations in areas subject to frequent winter road closures.

\subsubsection{Pressurized Ion Chamber Network}

At this time the PIC network consists of 29 instruments deployed around the NTS: 19 at the CRMSs and 10 at other sites, as shown on Exhibit 4-6. Not shown on Exhibit 4-6 are another 10 PICs, located at the Bureau of Land Management's Remote Automatic Weather Stations; data from these instruments are planned to be incorporated into the PIC network data base beginning in 1993. The instrument is manufactured by Reuter-Stokes and comes in ACoperated or battery-operated models. Most of the network is on commercial power, but several PICs operate on batteries charged by a solar power unit. The detector is an 8-L spherical aluminum chamber filled with high purity argon at 25 atmospheres pressure. The paper and cassette tapes are changed weekly. The data are transmitted every 4 hours via GOES directly to CP-1 and from there to EPA EMSL-LV by dedicated telephone lines. If the gamma exposure exceeds the site-specific alarm threshold for two consecutive 1-minute measurements, the system transmits a string of nine consecutive 1 -minute values on an average of every three minutes (typically varies between 2 and 15 minutes). The site-specific alarm thresholds are set at twice the normal background exposure.

\subsubsection{Hydrological Monitoring Program}

\subsubsection{Analysis}

The 3.8-L (1-gal) samples collected at each sampling location are preserved with nitric acid and are analyzed by high-resolution gamma spectrometry. For the semiannual onsite collections and all offsite collections, one of the two 5n0-mL (1-pt) samples from one collection period is analyzed for tritium by the conventional method, and the other is used either as a duplicate or replacement sample. One of the $500-\mathrm{mL}$ (1-pt) samples from each of the monthly onsite collections and from the other collection period for the semiannual onsite and offsite locations is analyzed for tritium by an enrichment method (EPA, 1979). The MDC for this method is less than $10 \mathrm{pCi} / \mathrm{L}$. 


\subsection{Data Management}

Data generated by the environmental monitoring networks are maintained in four data base systems: radiochemical data, TLDs, internal dosimetry, and PICs. Each of the four data systems includes its own data base management system, hardware, software, and procedures. Data processing, storage, and reduction procedures are specific for each of these four systems. Similar or common data verification, validation, and analysis procedures are used for all data.

\subsection{Radiochemical Database}

All radiochemical data are stored in the Sample Tracking and Data Management System (STDMS), a custom-designed database management system which resides on a MicroVAX II at EPA EMSL-LV. The MicroVAX II is networked to a large cluster of other mainframe and MicroVAX computers which comprises the "EMSL VAX network". Tracking of samples is initiated with input of data from sample tags and field forms. Analysis data are entered after they have been generated and reviewed by the analyst and supenisor. Special software written in Fortran (referred to as "Chemistry Programs") is used for a majority of the radiochemical data reduction. The Chemistry Programs are used for calculating final data such as activity per unit volume, MDC, and 2-sigma er:or terms. Standard report routines permit tracking of samples awaiting analysis, lists of QAVC samples, and sample results in various formats.

Access to STDMS is controlled by the Access Control List, keyed to user identification. Different levels of access are assigned based on the needs of a particular individual. A limited number of personnel have the access level necessary to make changes in the data base; these are primarily the personnel responsible for data entry, programmers, and the data base manager. Other personnel may access the data base in a "read-only" manner; their access permits generation of specific reports, but does not permit any changes, additions, or deletions to be made.

Once data have been entered and validated, they are transferred from a "review" data base to a permanent data base, e.g., further changes may be made only by authorized personnel. Any discrepancies noted during data validation processes are recorded on a standardized form. The form is reviewed and signed by the data base manager and Branch Chief. Actual changes are made by the data base manager or the contractor responsible for programming and maintenance of STDMS.

\subsection{Thermoluminescent Dosimetry Database}

The TLD data base resides on a Digital Equipment Corp. MicroVAX II, directly connected to the two Panasonic TLD readers. Samples are tracked using field data cards and an issue data base tracking system incorporated into the reade! control software. On-line QAVQC samples are processed with every group of field-deployed dosimeters. These include irradiated controls, transit controls, and processing laboratory background TLDs. Two major software packages are utilized by the TLD network. The first, a proprietary package written and supported by International Science Associates, controls the TLD readers, tracks dosimeter performance, completes necessary calculations to determine absorbed dose equivalent, performs automated QAQC functions, and generates raw data files and reports. The second, locally developed, maintains privacy act information and the identifying data, generates reports in a number of predefined formats, and provides archival storage of TLD results dating to 1971. 


\subsection{Internal Dosimetry Database}

Internal dosimetry utilizes a Canberra Nuclear Data System ND9900 "Genie" gamma spectrometer for acquisition, display, analysis and storage of whole body and lung counting data and for storage of the final bioassay results. The system incorporates a Digital Equipment Corp. MicroVAX II with the standard VMS software plus proprietary software packages written and supported by Canberra Nuclear Data Systems. In addition to the gamma spectroscopy operations, the software performs and stores calibration procedures and monitors QAVC functions. The software packages calculate dose using ICRP-30 methodology and generate reports and plots of the data and results. Personal identification and Privacy Act data are also stored as part of these records.

Additional Privacy Act information and more detailed residence location and exposure history, results of radioanalysis of bioassay samples, and final internal dose information are recorded in the MicroVAX using proprietary software called "Data Flex" developed by Data Access Corp. This is interactive data base software and allows retrieval of information from many more parameters than the Canberra software is capable of providing. Entries in this data base are verified by the staff using logbooks, and MicroVAX-and STDMS-generated reports.

Subject confidentiality and data security are maintained. Data from occupationally exposed persons are handled according to established DOE privacy act procedures. All internal dose information is considered Medical Confidential. Backups and archival storage is on the hard disk of the MicroVAX and on magnetic tape and diskettes stored both in the whole body counting facility and in the NRD offices.

\subsection{Pressurized Ion Chamber Database}

The data received via the GOES telemetry system are stored in indexed files on a MicroVAX. Weekly averages are calculated using the four-hour averages from the telemetry data and are stored in Lotus tiles on a PC. These values are presented in weekly reports which are sent to the monitoring stations and to DOE. Hourly and weekly averages are calculated by using the five-minute averages from the magnetic tapes and are then stored in debase files on a PC. The five-minute averages are also stored on a PC. Data acquired from the telemetry system are compared to data acquired from the magnetic tapes. Any anomalies are reported to the monitoring technicians. 


\title{
SECTION II.7B
}

\section{Operational Area Monitoring Plan for the Long-Term Hydrological Monitoring Program Off The Nevada Test Site}

\author{
by \\ S. C. Black \\ Reynolds Electrical \& Engineering, Co. \\ and \\ D. J. Chaloud \\ Environmental Monitoring Systems Laboratory \\ U. S. Environmental Protection Agency
}

January 21, 1993 


\subsection{Effluents}

There are indications "inat tritium escaped from the LONG SHOT cavity soon after detonation of that test, but the concentration of tritium in water samples has been decreasing at a rate somewhat faster than would be expected from just radioactive decay, an indication of diffusion. There is no indication from groundwater monitoring that tritium is continuing to leak from the test cavity.

At the DRIBBLE site on the Tatum Salt Dome near Baxterville, Mississippi, disposal of drilling muds and fluids near surface ground zero (SGZ) resulted in tritium contamination of shallow groundwater onsite. This shallow water, between 1.2 and $3 \mathrm{~m}$ ( 4 and $10 \mathrm{ft}$ ) deep, and a surficial aquifer that is $9 \mathrm{~m} \mathrm{(30} \mathrm{ft)} \mathrm{deep} \mathrm{both} \mathrm{consist} \mathrm{of} \mathrm{non-potable} \mathrm{water,} \mathrm{and} \mathrm{the} \mathrm{tritium}$ ccncentration in them has decreased to less than the National Primary Drinking Water Regulations value of $20,000 \mathrm{pCi} / \mathrm{L}(740 \mathrm{~Bq} / \mathrm{L})$. There is no indication from ground and surface water monitoring that any radioactivity is presently escaping from the test cavity.

Since 1984 a monitoring well near the Project GASBUGGY ground zero (GZ) has yielded slightly (less than 3 percent of the Interim Primary Drinking Water Regulations ' elevated levels of tritium. Due to the proximity of the well to $\mathrm{GZ}$, documented evidence of corimunication between the test cavity and the Ojo Alamo sandstone aquifer (Peter and Bo',iman, 1970), and the remote possibility that fracturing around the test cavity extends to the (jjo Alamo sandstone (DOE, 1986), the possibility of effluent migration from the test civity cannot be discounted. However, ihe low concentrations preclude the necessity of developing an effluent monitoring plan. No effluent release has been detected at the other test sites.

\subsection{Environmental Surveillance Plan}

\subsection{Design Criteria}

\subsubsection{Data Quality Objectives}

Each sample of surface and ground water consists of two parts: a 3.8-L (1-gal) sample collected in a plastic bottle for gamma spectrometric analysis and $500-\mathrm{mL}(1 \mathrm{pt})$ samples collected in glass bottles for ${ }^{3} \mathrm{H}$ analysis. The accuracy of the analytical methods is as follows:

\begin{tabular}{|c|c|c|}
\hline Type of Analysis & $\operatorname{LLD}^{1,2}$ & Accuracy@95\% Confidence In erval ${ }^{2}$ \\
\hline \multicolumn{3}{|l|}{ Tritium Analysis } \\
\hline Conventional & 500 & $\pm 30 \%$ at 600 or $\pm 10 \%$ at 5000 \\
\hline Enrichment & 10 & $\pm 30 \%$ at 12 or $\pm 20 \%$ at 100 \\
\hline \multicolumn{3}{|l|}{ Gamma Emitters } \\
\hline Range 60 to $2000 \mathrm{keV}$ & 5 & $\pm 30 \%$ at 6 or $\pm 20 \%$ at 50 \\
\hline
\end{tabular}




\subsection{Amchitka Island Projects, Alaska}

\subsubsection{Survelliance System Design}

The original hydrologic sampling network on Amchitka was estahlished by the Palo Alto Lab oratories of Teledyne isotopes (Essington 1971). The background or control sampling locations are shown in Exhibit 4-3-1 and listed in Table 4-3-1 together with other sites that have been sampled since the LTHMP began. Sampling locations for LONG SHOT are shown in Exhibit 4-3-2 and listed in Table 4-3-2. Those for MILROW are shown in Exhibit 4-3-3 and listed in Table 4-3-3, and for CANNIKIN are shown in Exhibit 4-3-4 and listed in Table 4-3-4.

\subsection{Project DRIBBLE, Mississippi}

\subsubsection{Environmental Surveillance}

\subsubsection{Criteria}

Groundwater monitoring is the only requirement for surveillance of this test site. The high rainfall rate in this area produces wetlands, flowing streams, and shallow aquifers that are monitored in the LTHMP. The many groundwater aquiliurs at the Tatum Dome Site are shown in Table 4-6-1. Although improbable, the four tests conducted within the dome could have opened cracks for seepage of test-produced radioactivity. Another route of escape for the radioactivity in the cavity is through the emplacement holes and post-shot holes that penetrate the cavity if the plugging activities had not produced perfect seals. In either case, the radionuclide most likely to first appear outside the cavity is tritium.

\subsection{Project SHOAL, Nevada}

Project SHOAL was sponsored by the Department of Defense and the U.S. Atomic Energy Commission (AEC) as a part of the Vela Uniform Program. The objective of the Project SHOAL experiment was to determine the effects caused by detonation of a nuclear device in a seismically active area (AEC 1964b).

\subsection{Project GASBUGGY, New Mexico}

\subsubsection{Operational Activities}

GASBUGGY was detonated on December 10, 1967, at 1230 Mountain Standard Time. The

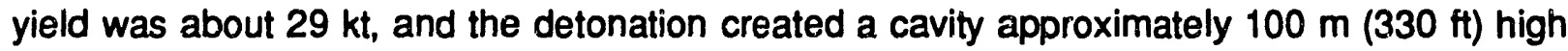
and $49 \mathrm{~m}$ (160 ft) in diameter. The PHS reported that its monitoring program detected no increase in radionuclides and no detectable tritium in surface and subsurface waters offsite. No fission products were detected in air samples and ground monitoring and personal dosimeters showed no increase over background levels. Post-test isotopic concentrations in milk samples were similar to those in pre-test samples (PHS 1970).

During the production test phase in June and July of 1968, in which the effect of the detonation on gas flow was determined, about $1000 \mathrm{Ci}$ of ${ }^{3} \mathrm{H}$ and $141 \mathrm{Ci}$ of ${ }^{85} \mathrm{Kr}$ were released to the atmosphere. Although extensive monitoring similar to that mentioned above was 
conducted, only samples of atmospheric moisture showed any detectable test-related radioactivity, i.e., ${ }^{3} \mathrm{H}$. The highest concentration detected was $1.2 \times 10^{-9} \mu \mathrm{Ci} / \mathrm{mL}$ in a sample collected on June 29 at $0.5 \mathrm{~km}(0.3 \mathrm{mi})$ from the release point. All releases were well documented and controlled, and no significant exposures to the general population occurred.

The only known effluents from this site occurred during the production test phases as summarized above. However, slightly elevated levels of tritium observed since 1984 in one well located near GZ may be indicative of fission product migration from the test cavity. These tritiumi levels have been less than 3 percent of the National Drinking Water Regulations. While continued surveillance is warranted, an effluent monitoring plan is not necessary at present.

\subsection{Data Management}

All radiochemical data are stored in the Sample Tracking and Data Management System (STDMS), a custom-designed database management system which resides on a MicroVAX II at EPA EMSL-LV. The MicroVAX II is networked to a large cluster of other mainframe and MicroVAX computers which comprises the "EMSL VAX network". Tracking of samples is initiated with input of data from sample tags and field forms. Analysis data are entered after they have been generated and reviewed by the analyst and supervisor. Special software written in Fortran (referred to as "Chemistry Programs") is used for a majority of the radiochemical data reduction. The Chemistry Programs are used for calculating final data such as activity per unit volume, MDC, and 2 standard deviation error terms. Standard report routines permit tracking of samples awaiting analysis, lists of QAQC samples, and sample results in various formats.

Access to STDMS is controlled by the Access Control List, keyed to user identification. Different levels of access are assigned based on the needs of a particular individual. A limited number of personnel have the access level necessary to make changes in the data base; these are primarily the personnel responsible for data entry, programmers, and the data base manager. Other personnel may access the data base in a "read-only" manner; their access permits generation of specific reports, but does not permit any changes, additions, or deletions to be made.

Once data have been entered and validated, they are transferred from a "review" data base to a permanent data base, e.g., further changes may be made only by authorized personnel. Any discrepancies noted during data validation processes are recorded on a standardized form. The form is reviewed and signed by the data base manager and Branch Chief. Actual changes are made by the data base manager or the contractor responsible for programming and maintenance of STDMS. 


\section{SECTION II.8}

\section{Operational Area Monitoring Plan for EG\&G/Energy Measurements, Inc. Support Facilities for the Nevada Test Site}

by

H. B. Gillen and J. W. Kesler EG\&G Energy Measurements, Inc.

January 22, 1993 


\subsection{Efíluents and Monitoring Plan}

\subsection{Monitoring Requirements for EG\&G/EM Operations}

\subsubsection{Effluent Monitoring Design Criteria}

\subsubsection{Standards, Criteria, and Action Levels}

The criteria for designing effluent monitoring programs are the applicable federal, state, and local regulatory requirements. These requirements are enforced for all EG\&G/EM operations primarily at the local level through a system of permits and notifications. The discharge standards, criteria, reference values, and/or action levels are listed or referenced in the discharge permits. Performance criteria for analytical procedures, e.g., probability of false positives or negatives, uncertainties, and statistical evaluation of analytical data are limited to that used by contract analytical laboratories performing analysis on the compliance monitoring samples.

\subsubsection{Monitoring Design Criteria}

Wastewater effluent from the RSL is discharged to the CCSDs POTW. The District has adopted Resolution No. 83-012 to regulate industrial users. Permit No. CCSD-032 was issued which establishes action levels and the standards and criteria for designing the monitoring system for this effluent. A copy of this permit can be found in Appendix II.8-B.

Wastewater from the NLV facility is discharged into the NLV POTW. The city has adopted ordinance number 730 to regulate industrial users. Permit No. 87-2 was issued with specific monitoring requirements for the printed circuit board and anodizing shop effluents. This permit establishes action levels and the standards and criteria for designing the monitoring system for these effluents. A copy of this permit and the associated conditions can be found in Appendix II.8-B.

\subsubsection{Wastewater Monitoring Systems}

The three regulated wastewater effluents at LVAO are from the printed circuit board and the anodizing shops (both at the NLV Facility) and the photography laboratory effluent at the RSL. However, there will be no further effluents from the printed circuit board plating operations which will need to be monitored. The printed circuit board plating operations have been permanently discontinued and the area shut down and completely decontaminated. Plating equipment has been disposed by various means.

\subsection{Amador Valley Operations}

\subsubsection{Monitoring Design Criteria}

The general goals, standards, and criteria for effluent monitoring are stated in Section 3.1.2. AVO does not have any water effluents that are subject to regulatory effluent monitoring requirements. Industrial wastewater discharge Permit No. 3672-101 issued by the Dublin San Ramon Services District, the local POTW, has been downgraded to a zero industrial wastewater discharge permit. A copy of this permit can be found in Appendix II.8-B. 
A permit application has been filed with the Bay Area Air Quality Management District for five small solvent cleaning operations. Effluent monitoring, if required, will be based on the requirements in the permit conditions once the permits have been issued.

\subsubsection{Effluent Monitoring System Design}

The effluent monitoring system for regulated effluents and at AVO must comply with permit conditions and address the general conditions stated in section 3.1.3. Since there are no regulatory requirments for monitoring AVO's effluents, there is no effluent monitoring system in place.

\subsection{Kirtland Operations}

\subsubsection{Effluents}

An inventory of effluents a . . source characterizations for the $\mathrm{KO}$ facility is contained in Table 3. KO has both a.v ain wastewater effluents. However, the effluents from KO are minimal, and discontinuous and are unregulated except for a permitted wastewater effluent at the Craddock facility.

\subsubsection{Monitoring Design Criteria}

The general goals, standards, and criteria for effluent monitoring are stated in Section 3.1.2. The only regulated effluent at $\mathrm{KO}$ is the rinse water from the alodining operation at the Craddock facility (see Table 3). This effluent is discharged into the local POTW. The regulatory authority for this effluent is the city of Albuquerque, Industrial Waste Engineer. Wastewater discharge Permit No. 2175A-R has been issued for this effluent by the City of Albuquerque which provides the criteria for designing the effluent monitoring system.

\subsubsection{Effluent Monitoring System Design}

The alodining operation has been permanently discontinued, effective August 31, 1992 which has eliminated the need for an effluent monitoring system for this operation.

\subsection{Santa Barbara Operations}

\subsubsection{Effluents}

An inventory of effluents and their source characterizations for the SBO facility are contained in Table 5. Based on the amounts of chemicals used, no air pollution control permits are required. SBO has two wastewater discharge permits issued by the Goleta Sanitation District; one for the 130 Robin Hill Road Facility and one for the 820 Francis Botello Road facility.

\subsubsection{Effluent Monitoring Design Criteria}

The general goals, standards, and criteria for effluent monitoring are stated in Section 3.1.2. SBO has two wastewater discharge permits issued by the Goleta Sanitation District. Industrial wastewater discharge Permit No. III-330 is for 130 Robin Hill Road Facility which includes the batch-treated wastewater from a mercuric iodide crystal process (see Table 5). Industrial 
wastewater discharge Permit No. All-204 is for the 820 Francis Botello Road facility which essentially has no industrial wastewater discharge. These permits establish the action levels, standards, and criteria for designing the monitoring system for this effluent. A copy of this permit can be found in Appendix II.8-B.

\subsubsection{Effluent Monitoring System Design}

The effluent monitoring system at SBO must comply with permit conditions and address the general conditions stated in Section 3.1.3. Although there is a limit to the concentration of mercury that can be discharged tc the sanitary sewer, no monitoring requirements are specified in Permit No. III-330. SBO does collect a sample from each treatment batch from the mercuric iodide crystal growing process to be picked up the Goleta Sanitation District (GSD) and analyzed for mercury at a GSD selected laboratory.

\subsection{Special Technologies Laboratory}

\subsubsection{Effluents}

An inventory of effluents and their source characterizations for the STL facility is contained in Table 6. STL has one air pollution control permit issued by the County of Santa Barbara, Air Pollution Control district for a vapor degreaser, and one industrial wastewater discharge permit for the STL facility.

\subsubsection{Effluent Monitoring Design Criteria}

The general goals, standards, and criteria for effluent monitoring are stated in Section 3.1.2. The criteria for designing effluent monitoring systems are the conditions of the permits issued to STL for regulated effluents. Authority to Construct Permit No. 8477 was issued for a small vapor degreaser which includes requirements for monitoring solvent use rates, recordkeeping and annual reporting. Industrial Wastewater Discharge Permit No. II-225 was issued for facility industrial wastewater. However, there are no monitoring requirments associated with this permit. A copy of these permits can be found in Appendix II.8-B.

\subsubsection{Effluent Monitoring System Design}

The effluent monitoring system for regulated effluents at STL must comply with permit conditions and address the general conditions stated in section 3.1.3. Presently the GSD analyzes the PC board rinse water at approximately annual intervals and assumes responsibility for compliance monitoring. Solvent use monitoring, recordkeeping, and annual reporting for the regulated vapor degreaser is managed by the site safety officer.

\subsection{Woburn Cathode Ray Tube Operation}

\subsubsection{Effluent Monitoring System Design}

The effluent monitoring system for regulated effluents and at WCO must comply with permit conditions and address the general conditions stated in section 3.1.3. No effluent monitoring 
is required for the trichloroethane degreaser, but if the annual use increases or a different solvent is used, then monitoring may be required.

The effluent wastewater passes through a limestone chip tank for $\mathrm{pH}$ adjustment, and samples for $\mathrm{pH}$ measurement must be taken before mixing with any other streams. Wastewater effluent flow rates are estimated semi-annually and samples taken and analyzed for $\mathrm{pH}$. Reports of monitoring activities are submitted semi-annually.

\subsection{Quality Assurance and Quality Control}

Quality Assurance (QA) is a system for ensuring that information, data, and resulting decisions completed under a specific task are technically sound, statistically valid, and properly documented. Quality Control (QC) comprises the controls implemented to ensure data quality.

The quality objectives for the existing effluent monitoring scheme are to demonstrate compliance and resolve issues of noncompliance through periodic sampling, emission calculations, reporting, and corrective actions for regulated effluents. Pursuant to: EG\&G/EM MANUAL NO. 14, QUALITY ASSURANCE, MANAGEMENT: POLICIES AND STANDARD OPERATING PROCEDURES; EG\&G/EM MANUAL NO. 15, QUALITY ASSURANCE PROGRAM REQUIREMENTS; EG\&G/EM MANUAL NO. 31, ENVIRONMENT, ENVIRONMENT, SAFETY AND HEALTH: POLICIES AND STANDARD OPERATING PROCEDURES; and the direction provided in the DOE/NV document, GUIDANCE FOR PREPARATION OF OPERATIONAL AREA MONITORING PLANS BY NTS CONTRACTORS, USERS AND SUPPORT AGENCIES, February 29, 1990, the general quality assurance procedures for data collection and management for monitoring regulated effluents are described below.

\subsection{QUALITY ASSURANCE MANAGEMENT}

The design of specific QA procedures for environmental monitoring is the responsibility of the Environmental Compliance Section at LVAO. The Safety Officer at each operation other than LVAO is responsible for the implementation of applicable QA procedures. For LVAO the Environmental Compliance Section is responsible for this implementation. The EG\&G/EM Environment, Safety and Health (ES\&H) Director has the primary oversight for the Environmental Compliance Programs including environmental monitoring activities for EG\&G/EM. See Appendix II.8C for organizational structures.

\subsection{DATA MANAGEMENT AND REPORTS}

This topic is covered in section 7.0 and 9.0 of this plan

\subsection{QA PROGRAM ASSESSMENT}

QA program assessment is the responsibility of EG\&G/EM Office of Quality Assurance. They set the schedule for performing periodic management and program audits which would include environmental monitoring activities. Their reports are sent to management. 


\subsection{PERSONNEL QUALIFICATIONS AND TRAINING}

Personnel qualifications for purposes of performing environmental monitoring activities is addressed in SOP No. 30-015.A, Qualifications of ES\&H Management and Prof. Personnel. Persons involved in environmental monitoring activities other than the individuals authoring the procedures noted in section 3.1.3 of this plan, must be trained in the applicable environmental monitoring procedures.

\subsection{LABORATORY SERVICES}

QA requirements are specified for all laboratory contracts pursuant to EG\&G/EM, Standard Operating Procedure No. 14-041.A, Establishing Procurement Quality Assurance.

Furthermore, contract laboratories must be vendor qualified in accordance with $E G \& G / E M$ Standard Operating Procedure No. 14-072.A, Supplier Qualification.

\subsection{DATA COLLECTION AND MANAGEMENT}

This section includes the data collection procedures to assure the quality goals are achieved. Environmental data collection activities include:

- Sample point selection

- Sampling method

- Documentation of samples and sampling activity

- Laboratory analyses, practices and procedures

- Emission calculations

The following quality assurance procedures are patterned after EPA guidance provided in the "Handbook for Sampling Water and Wastes."

\subsubsection{SAMPLE POINT SELECTION}

Unless otherwise specified in the permit conditions, the point at which the most representative sample of the regulated effluent can be taken shall be selected as the sampling point.

\subsubsection{SAMPLING METHOD}

\subsubsection{SAMPLING FREQUENCY}

The minimum sampling frequency for regulated effluents will be that specified in the effluent permit conditions.

\subsubsection{TYPE OF SAMPLE}

There are basically two types of samples that can be triken, each with their own list of variations. They are grab and composite samples.

A grab sample is defined as an individual discrete sample collected over a period of time not exceeding 15 minutes. It can be taken manually, using a pump, scoop, vacuum, or other suitable device. The collection of a grab sample is appropriate when it is desired to: 
- Characterize water quality at a particular time

- Provide information about minimum and maximum concentration

- Allow collection of variable sample volume

- Corroborate composite samples

- Meet a requirement of a discharge permit

A composite sample is defined as a sample formed by mixing discrete samples taken at periodic points in time or a continuous proportion of the flow. The number of discrete samples which make up the composite depends upon the variability of pollutant concentration and flow. Types of composite samples are:

- Continuous: constant pumping rate over a period of time

- Continuous flow proportioned: pumping rate is proportional to stream flow

- Periodic: constant sample volume, constant time interval between samples

- Periodic flow proportioned: constant sample volume, time interval between samples proportional to stream flow

The sample type selected must always be that which is specified in the permit. However, if the permit does not specify the sample type, then consider the following guidelines when selecting the sample type. A grab sample should be used when:

- The stream does not flow continuously such as batch dumps

- The water or waste characteristics are relatively constant

- The parameters to be analyzed are likely to change with storage such as dissolved gasses, residual chlorine, soluble sulfide, oil and grease, microbiological parameters, organics, and $\mathrm{Ph}$

A composite sample should be used when:

- Determining average concentrations

- Calculating mass/unit time loading

The ultimate goal is to secure a sample that is representative of the effluent. In addition, samples should be of sufficient volume to allow duplicate analyses and quality assurance testing (split or spiked samples) by the analytical laboratory. The required sample volume is a summation of that required for the analysis of each parameter of interest plus QC analyses. Sampling shall also be conducted in such a manner as to protect the integrity of the sample and prevent the inadvertent introduction of contaminants or foreign material.

\subsubsection{SAMPLE MANAGEMENT}

Sample size, containers, handling, preservation, and holding times shall be consistent with the requirements in 40 Code of Federal Regulations (CFR) Part 136, Test Procedures for the Analysis of Pollutants for wastewater effluent monitoring, unless otherwise specifically allowed by the appropriate regulatory authority, and Test Methods For Evaluating Solid Waste:

Physical/Chemical Methods, SW 846 for monitoring solid wastes as defined by the Resource Consenvation and Recovery Act. 


\subsubsection{WASTEWATER SAMPLING EQUIPMENT}

When wastewater sampling equipment is needed for composite sampling it shall be equipped with a variable speed peristaltic pump or an ISCO type periodic sampler, capable of taking composite samples of volumes not less than 2.5 gallions per 24 hours at remote locations. The wastewater sampling equipment must be rinsed before and after each sampling event with uncontaminated water. Care must be taken to insure the sampling equipment is compatible with the media being sampled and that it does not introduce contaminants into the sample.

\subsubsection{QUALITY CONTROL PROCEDURES FOR SAMPLING}

Various types of control checks should be randomly used to verify the sample collection and handling process has not affected the quality of the samples and validate the analytical laboratory's practices and procedures.

The two types of Quality Control blanks that can be periodically utilized are: trip blanks and equipment blanks. A trip blank is used to estimate sample contamination from the container and preservative during transport and storage of the sample. A cleaned sample container is filled with uncontaminated water; any preservative used in the sample is added to the blank in the same proportions as the real sample ; the blank is then stored, shipped, and analyzed with its group of samples. This blank is more useful when samples are not analyzed for several days or weeks because leaching of the material from the container can become significant. Contaminants found in the trip blanks could be attributed to: (1) interaction between the sample and container; (2) contaminated rinse water; (3) handling procedures that alter the sample analysis results or; (4) laboratory practices.

Equipment blanks are used to estimate incidental or accidental contamination of a sample during sample collection. To ensure that the non-dedicated sampling device has been effectively cleaned (in the laboratory or field), the device must be triple rinsed with deionized water. The final rinse water is collected in an appropriate container and shipped with the sample to the laboratory for analysis. Proper sample preservation and holding times must be observed.

Split samples can be used to validate the laboratory's analytical data. The original sample is split into two samples using identical containers and preservatives and should be shipped together to the laboratory under different identification numbers or to a different laboratory.

\subsubsection{DOCUMENTATION OF SAMPLES AND SAMPLING ACTIVITY}

\subsubsection{SAMPLE CONTAINER LABELS}

To adequately identify the sample, a sample container labels shall be filled out with indelible ink and include the following information:

- Name of sample point

- Date and time of collection

- Preservative used

- Unique sample identification number

- Analysis requested 


\subsubsection{SAMPLE CONTAINER SEALS}

Whenever monitoring is being conducted to resolve discrepancies, issues of noncompliance, or gather evidence for pending litigation, sample container seals must be used in conjunction with a chain of custody record. Samples must be sealed immediately after collection. Gummed paper seals are acceptable, as long as they only come into contact with the sample container and not the sample. Seals must be attached in such a way that they must be broken in order to open the sample container. The seal must include the following information, recorded with indelible ink:

- Sample ID Number

- Signature of the collector

- Date of sample

\subsubsection{SAMPLE LOGS}

A sample log shall be kept and include the following sampling information and recorded in indelible ink:

- Unique sample identification number

- Date and time of sample (on and off times)

- Description of the sample (include name, location and sample type) and method of sampling (grab or composite)

- Type of sample preservation used and holding times

- Name of the laboratory doing the analysis, the parameters of interest, and the analytical methods

- Name of collector

- Pertinent field data, e.g., pH

- Chain of custody used (yes or no)

- Field analysis

These records must be kept in a bound, paginated notebook. Errors made in entering information must not be erased or blotted out, but corrected by drawing a single line through the error and recording the correct information. Initialize and date the correction.

\subsubsection{CHAIN OF CUSTODY RECORD AND PROCEDURES}

Chain of custody procedures and records shall not be required for routine effluent monitoring activities for permit compliance. Chain of custody procedures and records shall be used when effluent monitoring activities are conducted to resolve discrepancies, issues of noncompliance, or gather evidence for pending litigation. This process is designed to establish the documentation necessary to trace sample possession from the time of collection through the laboratory analysis. When transferring the possession of the samples, the transferee shall sign and record the date and time on the chain of custody record. Custodial transfers should account for each individual sample, although samples may be transferred as a group. Every person who takes custody of a sample shall fill in the appropriate section of the Chain of Custody Record. As few custodians as possible should be in the chain of possession. Laboratory personnel are responsible for the care and custody of the sample once it is received by them. They should be prepared to testify that the sample was in their possession, in view, or secured in the laboratory at all times from the moment it was received from the custodian until time the analyses are completed. A sample is in someone's custody if it is in a 
person's actual physical possession, or first in a person's physical possession then kept within that person's view, or locked up, or kept in a secured area restricted to authorized personnel.

The field custodian is responsible for proper packaging and delivering samples to the appropriate personnel for shipment to the analytical laboratory. This responsibility includes completing the appropriate portion of the Chain of Custody Record and signing it.

All packages shipped to the analytical laboratory shall be accompanied by the Chain of Custody Record and other pertinent forms. A copy of each form should be retained by the facility of origin.

Mailed packages shall be registered with return receipt requested. If packages are sent by common carrier, receipts must be retained as part of the permanent chain-of-custody documentation. Procedures shall be developed to ensure the above documentation requirements are implemented.

Samples to be shipped must be packed to prevent breakage and the package sealed or locked, so that any evidence of tampering may be readily detected. Procedures shall be developed to ensure the samples are packaged and shipped accordingly.

\subsubsection{LABORATORY PRACTICE, ANALYSIS AND CERTIFICATION}

\subsubsection{LABORATORY PRACTICE}

Upon receiving the sample, the laboratory should verify the integrity of the sample. The sample should be inspected to see that:

- The sample is clearly marked and dated

- The sample was collected in an appropriate container

- The sample is properly preserved

- There is sufficient sample volume to perform all of the required analyses

- The sample is received in good condition and the custody seal (if used) is intact

- Chain of Custody Records match the number and description of samples

- Samples are analyzed within the proper holding times

- Samples requiring refrigeration are stored appropriately until the analysis is performed

\subsubsection{ANALYTICAL. PROCEDURES}

Any laboratory performing analysis of compliance samples must follow approved EPA analytical procedures as specified in the references noted at the end of this plan, or unless otherwise allowed by the regulatory agency requiring the analysis.

\subsubsection{LABORATORY CERTIFICATION AND QAQC PROGRAMS}

Contract laboratories performing compliance analyses shall be appropriately certified by the state of residence or otherwise approved by the regulatory agency requiring the analysis. It must also have a QAVC program in place that is acceptable to the state or agency issuing the certification. Each laboratory must ensure the following actions are completed:

- Calibration of laboratory instruments to within acceptable limits according to EPA or manufacturer's specifications before, after, and during use. Reference standards must be used when necessary. Records of calibrations must be available 
- Periodic inspection, maintenance, and servicing (as necessary) of all laboratory instruments and equipment

- The use of reference standards and quality control samples (e.g., checks, spikes, laboratory blanks, duplicates, or splits), as necessary, to determine the accuracy and precision of procedures, instruments, and operators

- The use of adequate statistical procedures (e.g., quality control charts) to monitor precision and accuracy of the data and to establish acceptable limits

- A continuous review of results to identify 2 , correct problems within the measurement system (e.g., instrumentation problems, inituequate operator training, inaccurate measurement methodologies)

- Documentation of the performance of systems and operations

- Regular participation in external laboratory evaluations to determine the accuracy and overall performance of the laboratory. This should include performance evaluation, interlaboratory comparison studies, and formal field unit/aboratory evaluations and inspections

\subsubsection{AIR EMISSION CALCULATIONS}

If air emission calculations are required as a condition of a permit, then they may be done using any combination of the following methodologies: operational data provided by the process supervisor or operator of the emission unit, emission factors, or analytical data from source testing. Allowable emission factors are those derived from Material Safety Data Sheets, manufacturer factors for process equipment and efficiency ratings of air pollution control equipment, EPA emission factors and emission factors provided by the regulatory authority.

\subsection{DISCREPANCIES AND CORRECTIVE ACTION}

The analytical laboratory performing analyses on samples taken for compliance monitoring shall be responsible for resolving its own discrepancies and taking corrective action when predetermined limits for data analytical data acceptability are exceeded.

Each operation shall be responsible for resolving its own discrepancies and taking corrective action as a result of environmental appraisals, QA audits, or quality control checks.

Procedures for addressing discrepancies shall be included in the required effluent monitoring procedures (see section 3.1.3 of this plan).

\subsection{INDEPENDENT DATA VERIFICATION}

Guidance on independent data verification will be provided by DOE/HQ. 


\title{
SECTION III
}

\section{Groundwater Monitoring Plan for the Nevada Test Site}

\author{
by \\ R. Hershey \\ Desert Research Institute \\ University of Nevada System
}

January 21, 1993 


\subsection{Introduction}

\subsection{Groundwater Protection Management Plan}

The present version of the Groundwater Monitoring Plan (GMP) was written specifically to address possible future groundwater monitoring activities at the NTS after completion of a Remedial Investigation/Feasibility Study for underground nuclear testing sites. As such, it does not meet the complete requirements for a GMP as specified by DOE Order 5400.1. The 1993 revised GMP will comply with the Order and will include information on three major groundwater programs at the NTS, Groundwater Characterization, Groundwater Monitoring and Groundwater Protection. A brief description of these programs is presented below.

Groundwater characterization activities are performed in several different areas including, Environmental Restoration (ER), Area 5 Radioactive Waste Management Site Characterization, and the Hydrology/Radionuclide Migration Program (HRMP). Groundwater characterization activities in the ER program are performed in compliance with CERCLA which requires characterization of potential contamination sources and transport of those contaminants by groundwater for determination of risk to human health and the environment. To satisfy these requirements, drilling of approximately 100 new wells and refurbishing of other existing wells is in progress. Groundwater and unsaturated-zone characterization activities are being performed at the Area 5 Radioactive Waste Management Site to complete a RCRA Part B permit application. The HRMP is focused on the specific needs of the present and future weapons testing program with program activities including, long-range hydrologic studies and shot specific studies.

Groundwater monitoring is conducted at the NTS both for compliance with environmental laws and DOE orders and for management of water resources. Groundwater monitoring activities for environmental law and regulation compliance include, sampling of water-supply wells for Safe Drinking Water Act constituents, well installation and groundwater sampling to complete the RCRA Part B permit application for the Area 5 site, and sampling of non-routine effluents for RCRA-regulated contaminants. CERCLA driven activities are being conducted in anticipation of listing of the NTS on the National Priorities List defined in the National Oil and Hazardous Substances Contingency Plan. These CERCLA activities, which may include groundwater monitoring, are outlined in Section III of the Environmental Monitoring Plan. Groundwater monitoring is also conducted for water-resource management and groundwater stewardship. The Long-Term Hydrological Monitoring Program has been in operation since the 1960's and consists of routine sampling of wells on and around the NTS. Samples are analyzed for tritium and gamma activity. The analytical results are published annually in the DOE/NV Annual Site Environmental Report. The HRMP monitors water levels in wells on and around the NTS to produce current groundwater elevation maps and update groundwater flow models. The HRMP also monitors water-resource usage, conducts contaminant source characterization and migration studies, and monitors nuclear device emplacement-hole fluid levels and tritium activity.

Groundwater protection activities include, evaluating device emplacement-hole locations for potential impacts on groundwater, establishing environmental regulatory compliance of surface facilities, and incorporating waste minimization and pollution prevention awareness into daily activities. NTS-SOP-5417 defines five criteria for protection of groundwater during the citing of underground nuclear tests. Before an emplacement hole is drilled or an emplacement drift is mined, compliance with the criteria must be demonstrated by the 
sponsoring user and verified by a DOE review. Several environmental regulatory compliance programs including, Clean Water Act discharge permitting; Safe Drinking Water Act monitoring of water-supply wells, and implementing of a Wellhead Protection Program; RCRA permitting of new waste management activates, operations of existing waste management facilities, and closure of other waste management facilities; and, CERCLA characterization and remediation activities exist to provide groundwater protection from surface activities. 


\title{
SECTION IV
}

\section{Meteorological Monitoring Plan of the Weather Service Nuclear Support Office for the Nevada Test Site}

\author{
by \\ D. Randerson \\ Weather Services Nuclear Support Office \\ National Oceanic Atmospheric Administration
}

January 21, 1993 


\subsection{Meteorological Monitoring}

\subsection{National Weather Service Systems}

\subsubsection{Satellite Weather Information System}

WSNSO operates three SWIS systems. One is located in the main forecast office in the Las Vegas DOE building, one is at CP-1 in Area 6 on the NTS, and the third one is at the Desert Rock (DRA) WSMO. The SWIS in Las Vegas and at DRA are used for routine daily operations and are monitored 24 hours daily. The system at CP-1 is used to support nuclear test operations. 


\section{DISTRIBUTION LIST}

Manager Environmental Management Division, Reynolds Electrical \& Engineering Co., Inc., Post Office Box 98521, Las Vegas, NV 89193-8521

Information Products, Reynolds Electrical \& Engineering Co., Inc., Post Office Box 98521, Las Vegas, NV 89193-8521, MS 551 (2)

Office of Scientific and Technical Information, Technical Information Center, U.S. Department of Energy Post Office Box 62, Oak Ridge, TN 37831 (2)

William Wiggins, DOE Nevada Field Office, U.S. Department of Energy, Post Office Box 98518, Las Vegas, NV 89193-8518, M/S 505 (30)

Fred Ferate, Environmental Management Division, Reynolds Electrical \& Engineering Co., Inc., Post Office Box 98521, Las Vegas, NV 89193-8521, MS 776

Glen Clark, Reynolds Electrical \& Engineering Co., Inc., Post Office Box 98521, Las Vegas, NV 89193-8521, MVS 711

Scott Wade, Reynolds Electrical \& Engineering Co., Inc., Post Office Box 98521, Las Vegas, NV 89193-8521, MS 711

Gregory Shott, Reynolds Electrical \& Engineering Co., Inc., Post Office Box 98521, Las Vegas, NV 89193-8521, MS 738

Allan Smith, Ratheon Services Nevada, Post Office Box 95487, Las Vegas, NV 89193-5487, MS 424

R. L. Hershey, Desert Research Institute, 2505 chandler Avenue, Suite \#1, Lals Vegas, NV 89120

Deb Chaloud,U.S. Environmental Protection Agency, Post Office Box 93478, Las Vegas, NV 89193-3478, M/S 513

Richard W. Henderson, Los Alamos National Laboratory, Post Office Box 0, Mercury, NV 89023 MS 967

Ken Raschke, Lawrence Livermore National Laboratory, Post Office Box 45, Mercury, NV 89023, M/S 777

Darryl Randerson, U.S. Department of Commerce/Weather, Service Nuclear Support Office, Post Office Box 14985, Las Vegas, NV 89114, M/S 516

Harold Gray, EG\&G Energy Measurement Group Inc., Post Office Box 1912, Las Vegas, NV 89125 MS 570/L-01 
Bruce Gillen, EG\&G Energy Measurement Group Inc., Post Office Box 1912, Las Vegas, NV 89125 M/S 570/L-01

James Metcalf, Sandia National Laboratories, Post Office Box 238, Mecury, NV 89023, M/S 944

Dave Bedsun, Defense Nuclear Agency, Post Office Box, 98539, Las Vegas, NV 89193-8518, MS 573 

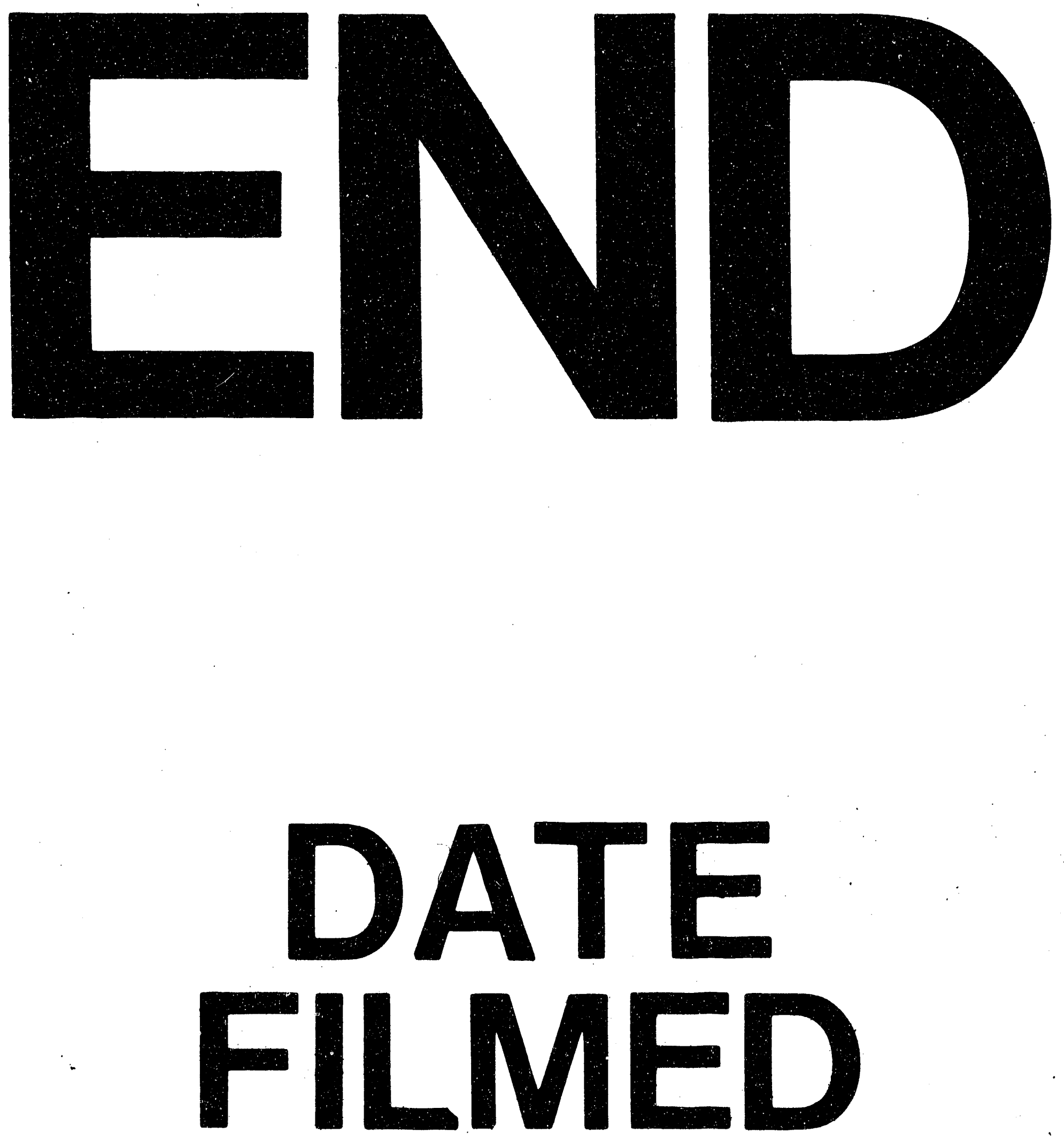

I

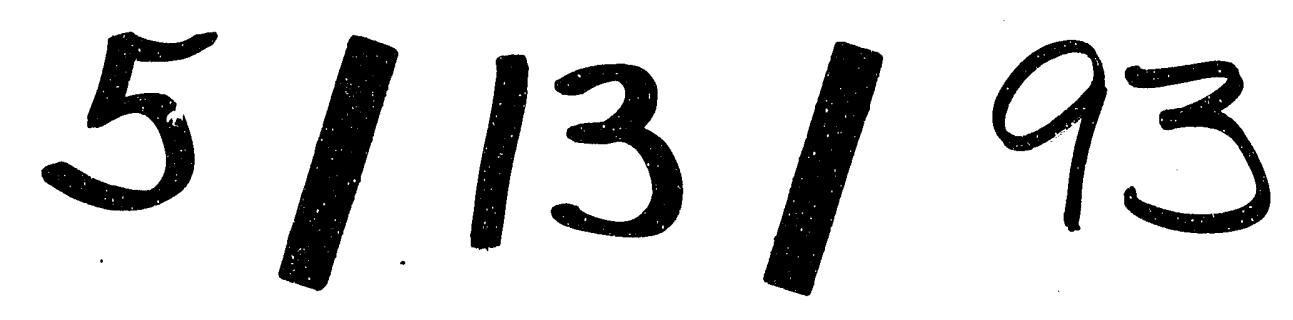


